\title{
Virtual Reality Representations of Nature to Improve Well-Being amongst Older Adults: a Rapid Review
}

\author{
Josca Van Houwelingen-Snippe ${ }^{1}$ (i) . Somaya Ben Allouch ${ }^{2}$. Thomas J. L. Van Rompay ${ }^{1}$
}

Received: 15 July 2020 / Revised: 19 January 2021 / Accepted: 25 January 2021 / Published online: 5 March 2021

(c) The Author(s) 2021

\begin{abstract}
Poor well-being amongst older adults poses a serious health concern. Simultaneously, research shows that contact with nature can improve various facets of well-being, including physical, social, and mental well-being. However, nature is not always accessible for older adults due to mobility restrictions and related care needs that come with age. A promising strategy aims at bringing nature inside through pervasive technologies. However, so far, there is little academic understanding of essential nature characteristics, psychological processes involved, and means for implementation in practice. The current study used a three-folded rapid review to assess current understanding and strategies used for improving well-being for older adults through virtual reality representations of nature. Searches were performed across three databases, followed-up by contentbased evaluation of abstracts. In total, a set of 25 relevant articles was identified. Only three studies specifically focus on digital nature as an intervention strategy for improving well-being amongst older adults. Although these studies provide useful starting points for the design and (technological) development of such environments, they do not generate understanding of how specific characteristics of virtual nature representations impact social well-being measures in particular, and of the underlying psychological processes involved. We conclude that follow-up research is warranted to close the gap between insights and findings from nature research, gerontology, health research, and human-technology interaction.
\end{abstract}

Keywords People-environment interaction · Digital nature · Social well-being $\cdot$ Connectedness $\cdot$ Human-technology interaction

\section{Introduction}

Poor well-being amongst elderly is increasingly recognized as a serious public health concern (Cacioppo et al., 2017; Hawkley \& Cacioppo, 2007; Sorkin et al., 2002). Wellbeing comprises several domains, including physical, mental, social, and economic well-being (OECD, 2013). Research shows that interaction with nature can improve a wide range of well-being facets including happiness, positive affect, feelings of connectedness, and a sense of meaning in life (Bratman et al., 2019; Kaplan \& Kaplan, 1989;

Josca Van Houwelingen-Snippe

j.vanhouwelingen-snippe@utwente.nl

$\bowtie$ Somaya Ben Allouch

s.ben.allouch@hva.nl

1 University of Twente, Enschede, The Netherlands

2 Amsterdam University of Applied Sciences, Amsterdam, The Netherlands
Maas et al., 2009; van Houwelingen-Snippe et al., 2020a, b). However, nature is often not accessible for older adults because of mobility issues or because nature is becoming increasingly scarce in urbanized regions. Interestingly, recent developments underscore the potential of modern technologies including virtual and augmented reality for bringing nature inside. At the same time, research shows that indirect encounters with nature (such as watching videos of nature scenes) can already improve well-being (Baños et al., 2012). Taking note of advances in technology development and recent research findings from the social sciences, this review aims at identifying research studying the interplay between digital nature and well-being amongst older adults.

\section{Well-Being amongst Elderly}

Poor well-being poses a serious public health concern (Cacioppo et al., 2017). Poor well-being may lead to mental health issues (i.e., depression, loneliness, and mood 
disorders) and physical health issues, including greater risk of cardiovascular disease (Sorkin et al., 2002) and accelerated physiological decline (Hawkley \& Cacioppo, 2007). There is a long debate going on in the literature about the conceptual clarity of the concept of well-being. In this study, we define well-being as a complex, multilevel and multidimensional concept in which well-being is regarded as a state of equilibrium between elements within the body (e.g., bodily rhythms and processes) and external influences operating outside the body (e.g., social context, atmosphere, and the physical environment). Hence, well-being is a dynamic process that is affected by life events and (social) challenges (e.g., shrinking networks that come with old age) that humans continuously face (Dodge et al., 2012; Ng \& Fisher, 2013; Fiorini et al., 2016; OECD, 2019a). In general, people experience high levels of well-being when they have the resources needed to meet and manage life's challenges (Dodge, et al., 2012; Fiorini et al., 2016).

\section{Nature and Well-Being}

A growing body of literature underscores the positive effects of nature experience on well-being, as also evidenced by several systematic reviews (Annerstedt \& Währborg, 2011; Bratman et al., 2019; Gascon et al., 2015; Hunter et al., 2019). For instance, urban green space interventions can improve health and social benefits (Hunter et al., 2019) and being close to, or living in, nature can also reduce feelings of loneliness and boost perceptions of social support (Maas et al., 2009). Other reviews (Bratman et al., 2019; Gascon et al., 2015) point out that research is needed to identify causal links between nearby green space and (mental) well-being (i.e., what are underlying mechanisms and key properties of nature spaces that promote better mental health?), and to clarify the relationship between exposure duration and frequency of visits and effects obtained (Gascon et al., 2015). A cross-disciplinary body of evidence (including research from social and health sciences) stresses the importance of nature experience on mental well-being (Bratman et al., 2019). Based on this evidence, a conceptual model is presented to disseminate insights amongst stakeholders (such as city planners or architects) in order to raise awareness of the impact of urban planning decisions on mental well-being (Bratman et al., 2019).

In line with findings from these reviews, it has been shown that nature-based therapies (e.g., horticultural or wilderness therapy) can be effective and may complement therapy programmes for a variety of mental and physical diagnoses, such as dementia and obesity (Annerstedt \& Währborg, 2011). Concluding, a considerable body of research documents the link between contact with nature (and related dimensions such as accessibility and availability of nature) and well-being. In the next section, studies focusing on elderly and nature interaction will be discussed.

\section{Elderly and Nature}

Nature interaction seems to be beneficial for everyone. However, contact with nature and the close proximity of nature play a particularly important, yet nuanced, role in older adults' everyday life (Finlay et al., 2015). By consequence, a relatively large body of research focuses on well-being benefits of nature for older adults (Detweiler et al., 2012; Kabisch et al., 2017; Wen et al., 2018).

In general, older adults benefit from green space as illustrated by a positive association between the availability of green space and perceived general health (Kabisch et al., 2017). According to a systematic review (including 44 articles) on the needs and preferences of older adults (Wen et al., 2018), older adults who engage in recreational activities in green spaces particularly value naturalness, aesthetics, and variety within the scene. Furthermore, for logistical reasons and safety considerations, accessibility of the green space and the inclusion of well-maintained paths are crucial for older adults to enjoy nature's benefits (Wen et al., 2018). In line with the importance of being active in nature, therapeutic gardens and horticultural therapy have also been pointed out as particularly suited to older adults in general (Detweiler et al., 2012; Milligan et al., 2004), and to people living with dementia in particular (Hernandez, 2007; Murphy et al., 2010).

Various reviews have been undertaken focusing on social well-being, and loneliness in particular, amongst older adults (Landeiro et al., 2017) and possible interventions for reducing it (Dickens et al., 2011a; Fakoya et al., 2020). Factors predicting loneliness are widowhood, older age, poor mental or physical health, and being new in a community (De Koning et al., 2017). The experience of loneliness varies greatly across individuals, which makes it extremely challenging, if not impossible, to design a one size fits all loneliness intervention, according to a recent scoping review on 33 review articles (Fakoya et al., 2020). According to another systematic review (Dickens et al., 2011b), successful and effective interventions targeting social isolation share three characteristics: theory-informed (i.e., evidence-based) development, provision of social activity, and/or group support. Additionally, an active lifestyle also seems to increase effectiveness of interventions targeting social isolation in older adults (Dickens et al., 2011b). These research endeavours testify to the ongoing search for effective interventions promoting social well-being by decreasing loneliness and social isolation amongst older adults.

To sum up, we discussed a number of review articles focusing on the beneficial effects of nature on well-being (Annerstedt \& Währborg, 2011; Bratman et al., 2019; 
Gascon et al., 2015), on social well-being amongst elderly (De Koning et al., 2017; Dickens et al., 2011a; Fakoya et al., 2020; Landeiro et al., 2017) and on the importance of nature interaction for promoting well-being of older adults in particular (Kabisch et al., 2017; Wen et al., 2018). In the present review, we aim to identify articles that focus on the cross sections of these topics: the effects of nature interaction on well-being for older adults.

\section{Virtual Reality Representations of Nature}

Older adults do not always have access to nature and hence cannot enjoy nature's benefits. When considering how to make nature accessible for people with limited or no access to nature, studies looking into the effects of virtual reality representations of nature are of particular interest. Research on the comparison of real-life nature interaction and virtual nature interaction indicates that simulated nature may exert similar benefits when compared with real nature (Annerstedt et al., 2013; Browning et al., 2020; Kjellgren \& Buhrkall, 2010). Promising examples in health care research are augmented biking exercises with augmented nature (BruunPedersen et al., 2014; Bruun-Pedersen et al., 2016; Grani \& Bruun-Pedersen, 2017) or virtual nature in nursing homes for recreational purposes (Bruun-Pedersen et al., 2015a; Ludden et al., 2019). In short, these combined findings underscore the potential of virtual nature for enhancing diverse facets of well-being.

In the review study described next, we performed three rapid reviews to identify existing studies investigating the effects of digital nature on well-being for elderly. There has been considerable attention for the individual topics under investigation, and even for cross-topic combinations (e.g., assistive technology to reduce loneliness amongst older adults [Jansen-Kosterink et al., 2018; Ring et al., 2013; Ten Bruggencate et al., 2018; Zamir et al., 2018]). However, in this review, we are specifically interested in multi-disciplinary research aimed at integrating findings from nature studies, human technology interaction, and social and health-related studies. Therefore, the aim of the present review is to identify articles that focus on the effects of nature interaction on well-being for older adults. On top of that, the second aim of this review is to identify articles focussing on virtual reality representations of nature for older adults as a means to improve social well-being in particular. On the one hand, there are many creative technology applications and initiatives aimed at implementation of virtual reality representations of nature in various care settings. However, most of these are not evidence or theory-based. On the other hand, studies from the social sciences are revealing about the psychological processes involved. However, these studies usually do not aim at facilitating the bridge from science to practice. Combining these disciplines will open up new possibilities for health innovations.

\section{Methods}

\section{Search Strategy}

A rapid review was performed, to assess what is already known about Well-being, Elderly, Technology and Nature. A rapid review method is one of the review methods which fall under the umbrella of Cochrane Review Methods (Moher et al., 2015; Garritty et al., 2016). A rapid review has been described as evidence synthesis that uses methods to streamline those of systematic reviews to complete the evidence synthesis in a shorter turnaround time than a standardized systematic review (Gannan et al., 2010; Khangura et al., 2014; Polisena et al., 2015). Furthermore, a rapid review follows many of the principal steps of a systematic review, using systematic and transparent methods to identify, select, and critically analyse data from the relevant databases but the main difference is that some of the elements of a rapid review are either simplified or omitted, such as for example using one reviewer or reducing the number of used databases (HEARD, 2018). For this study, we used the online databases Scopus, Web of Science, and PubMed and did not include for example the IEEE database. Only studies written in the English language were considered.

\section{Search Part 1}

The search key words used in the study are presented in Table 1 arranged per topic.

For all databases, all combinations of the search key words were used. All synonyms per topic were connected with a disjunction ("elderly" OR "aged" OR "older" etc.) and all topics were connected with a conjunction (Elderly (and all synonyms) AND Technology (and all synonyms) etc.).

A content analysis was performed on those articles that were selected based on the combination of all topics (Elderly and Nature and Well-being and Technology). Two reviewers performed the search and reviewed the selected articles. Each reviewer decided whether (1) each abstract concerned the experience of [or interaction with] nature, (2) whether the study used digital representations of nature, and (3) whether the focus was on (a dimension of) well-being. Articles that described participant groups which included participants aged 65 and older were included. 
Table 1 Search key words per topic Search Part 1

\begin{tabular}{|c|c|c|c|}
\hline Elderly & Technology & Well-being & Nature \\
\hline "Elderly" & Technolog* & "Wellbeing" & "Nature experience" \\
\hline "Aged" & "System" & "Well-being" & "Nature exposure" \\
\hline "Older" & "Virtual Reality" & "Positive mental wellbeing" & "Restorative nature" \\
\hline "Elder" & "VR" & "Positive mental well-being" & "Nature environment" \\
\hline "Geriatric" & "Augmented reality" & "Subjective wellbeing" & "Green space" \\
\hline "Elderly people" & "Ambient technology" & "Subjective well-being" & "Blue space" \\
\hline "Old people" & "Pervasive technology" & "Psychological wellbeing" & \\
\hline \multirow[t]{12}{*}{ "Senior" } & & "Psychological well-being" & \\
\hline & & "Emotional wellbeing" & \\
\hline & & "Emotional well-being" & \\
\hline & & "Social connectedness" & \\
\hline & & "Social isolation" & \\
\hline & & "Social wellbeing" & \\
\hline & & "Social well-being" & \\
\hline & & "Connectedness" & \\
\hline & & "Loneliness" & \\
\hline & & "Social isolation" & \\
\hline & & "Mental health" & \\
\hline & & Psycholog* & \\
\hline
\end{tabular}

\section{Results Part 1}

The number of unique papers selected from the databases was 100 (see Appendix 1 for a table comprising all studies). A content evaluation of the abstracts of the selected papers was performed (see Fig. 1 for the selection process).

Cohen's $\kappa$ was determined to assess level of agreement between the two reviewers. Specifically, the 100 papers and abstracts were assessed based on the three criteria outlined above (i.e., whether they concerned experience or interaction with nature, used digital representations of nature, and centred on a well-being-related outcome measure). Initially, the agreement between the two reviewers was moderate on the topic of nature interaction $(\kappa=.46)$, perfect on the topic of digital nature $(\kappa=1)$, and substantial on the topic of wellbeing $(\kappa=.67)$. After discussion between the reviewers, a substantial agreement on the topic of nature interaction $(\kappa=.78)$ and an excellent agreement on the topic of wellbeing $(\kappa=.86)$ was achieved. For the remaining 13 articles for which no agreement was reached, a third reviewer was involved to review these articles. Finally, agreement was achieved between the three reviewers, while the Cohen's kappa remained stable (nature interaction $(\kappa=.78)$, digital nature $(\kappa=1)$, well-being $(\kappa=.86))$.

\section{Study Characteristics}

Only articles that covered at least three of the topics, according to research aim 1, were included in the in-depth analysis. Table 2 presents an overview of the 27 articles included and their main findings.

It is important to note that although 27 studies were selected which met at least three of four criteria, only two studies met all four of the criteria and describe research on virtual reality representations of nature to improve wellbeing for older adults.

To sum up, the aim of this review was to identify current insights in studies on benefits of virtual reality representations of nature on well-being for elderly. Since

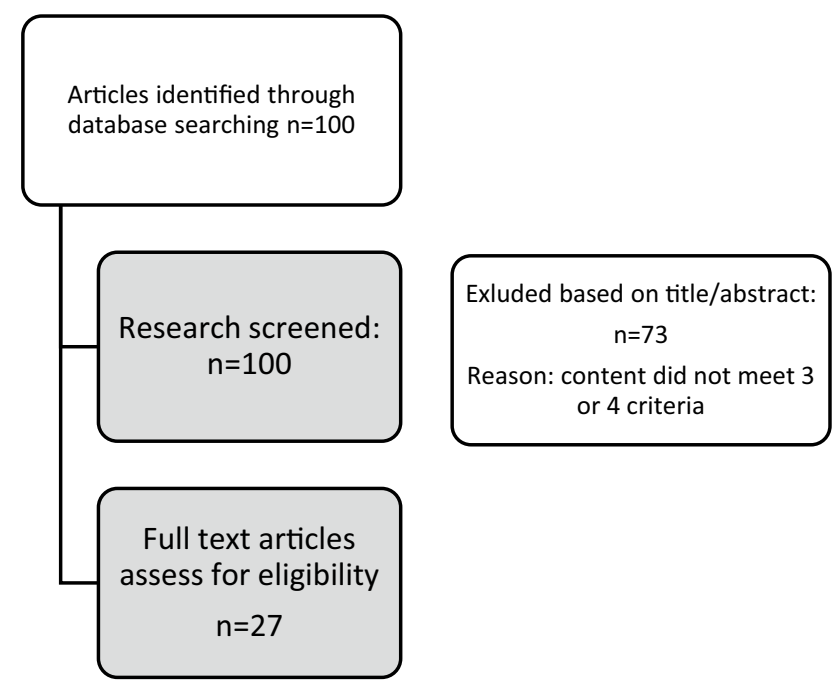

Fig. 1 Selection procedure Search Part 1 


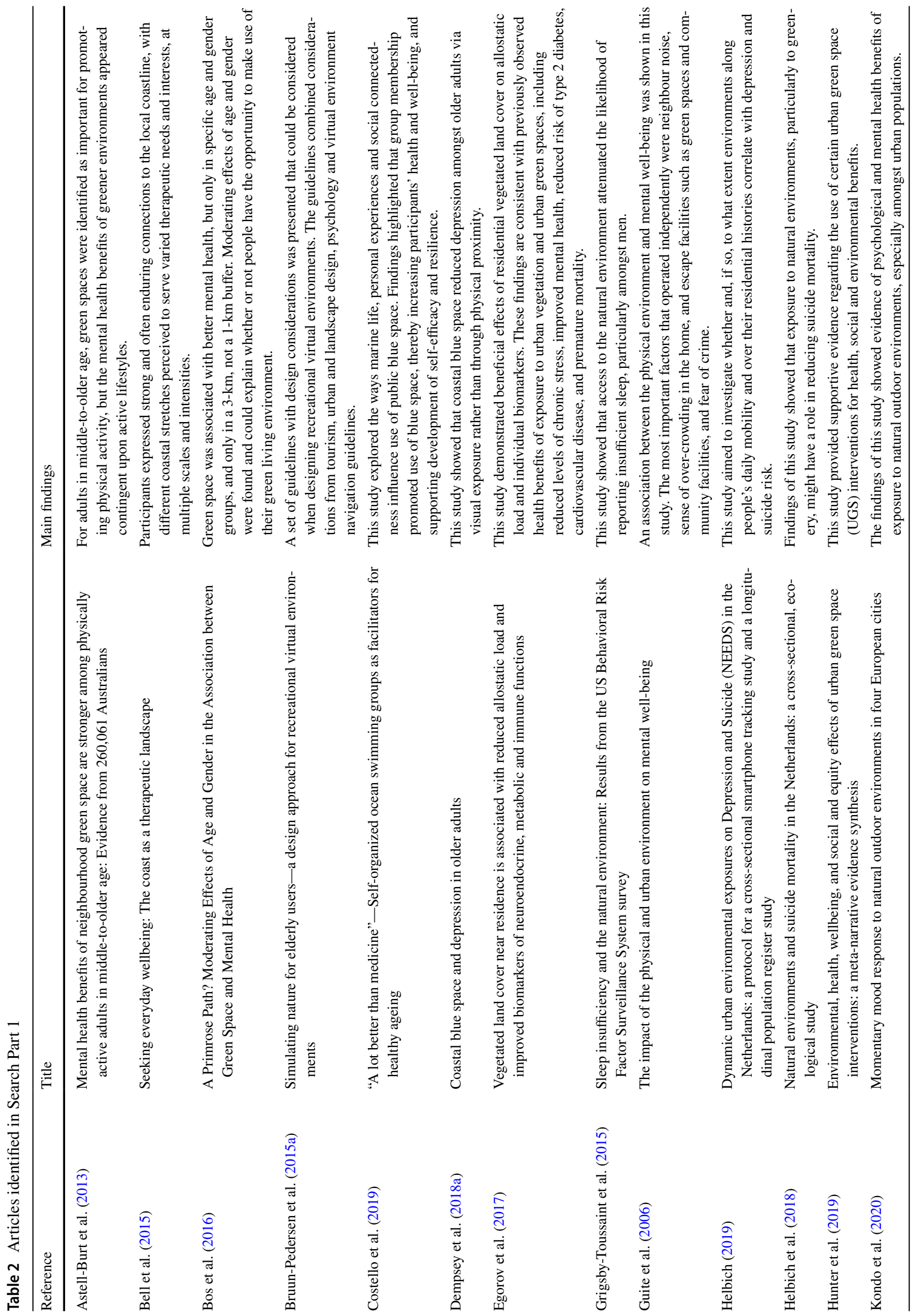




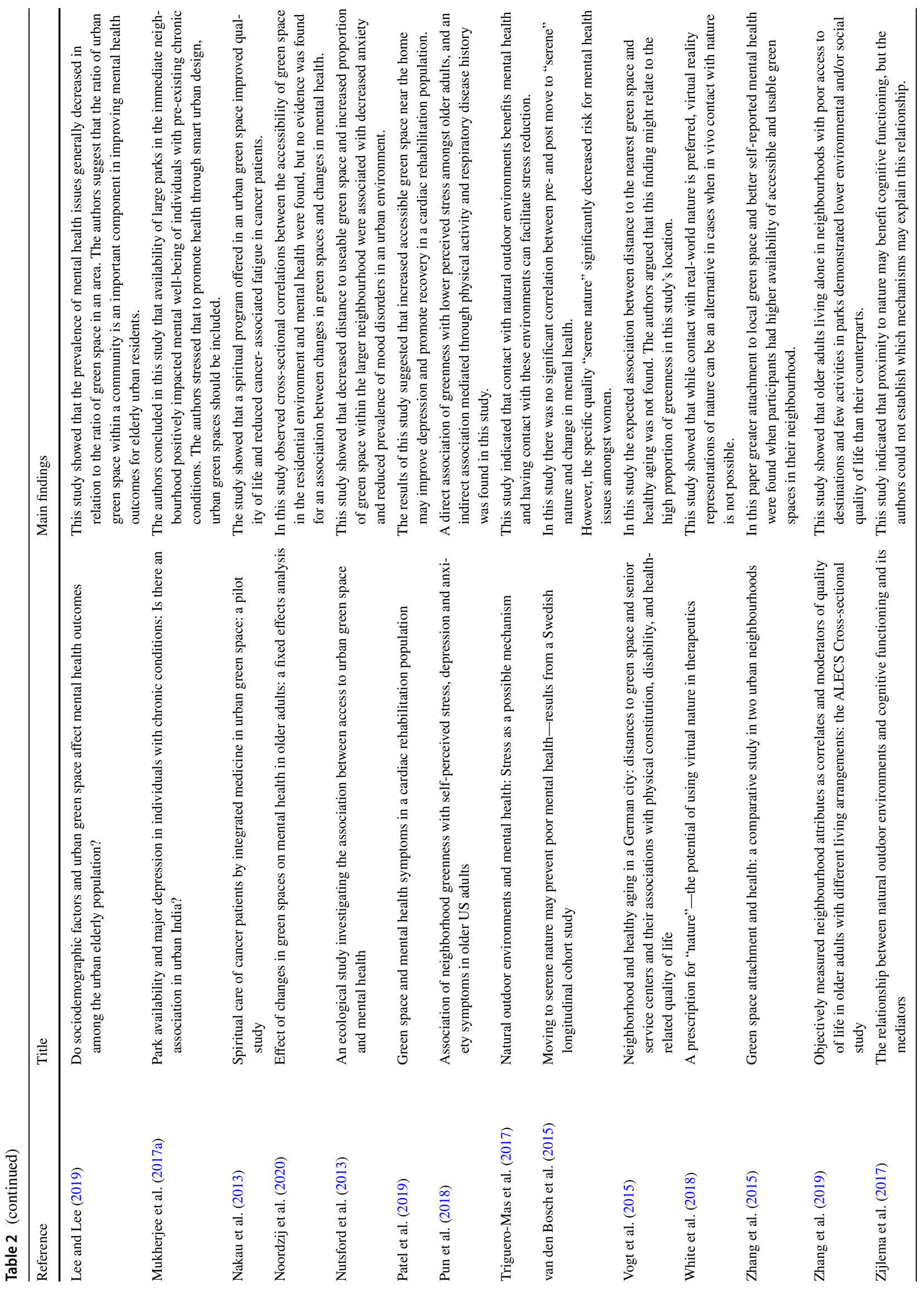


Table 3 Search key words per topic (Search Part 2)

\begin{tabular}{|c|c|c|c|}
\hline Elderly & Technology & Connectedness & Nature \\
\hline "Elderly" & Technolog* & "Social connectedness" & "Nature experience" \\
\hline "Aged" & System & "Connectedness" & "Nature exposure" \\
\hline “Older" & "Virtual reality" & "Loneliness" & "Restorative nature" \\
\hline "Elder" & "VR" & "Social isolation" & "Nature environment" \\
\hline "Geriatric" & "Augmented reality" & "Mental health" & "Green space" \\
\hline "Elderly people" & "Ambient technology" & Psycholog* & "Blue space" \\
\hline "Old people" & "Pervasive technology" & & \\
\hline "Senior" & & & \\
\hline
\end{tabular}

only 2 of the 27 selected papers actually met all four criteria, we feel safe to conclude that there is a lack of integration of insights across the four different topics. We decided to run a second search with a stronger focus on connectedness (rather than well-being) to identify relevant studies on social aspects of well-being missed in the first round.

\section{Search Part 2}

The search key words (arranged per topic) are presented in Table 3.

\section{Results Part 2}

We performed a content evaluation on the abstracts of the selected papers of the search combining all topics (Elderly $\&$ Nature \& Connectedness \& Technology). Figure 2 shows the selection process.

After checking for duplicates, 52 articles remained. A table representing all selected articles (and topics covered), that were not selected in part 1, is presented in Appendix 2.

\section{Study Characteristics}

Similar to part 1 , in search part 2 only studies that met at least three of the criteria were included in the in-depth analysis. Only one new study was identified (see Table 4); 16 studies were identified that were already discussed in Table 2 (Astell-Burt et al., 2013; Bos et al., 2016; BruunPedersen et al., 2015a; Dempsey et al., 2018b; Egorov et al., 2017; Grigsby-Toussaint et al., 2015; Helbich et al., 2018; Lee \& Lee, 2019; Mukherjee et al., 2017a; Nakau et al., 2013; Nutsford et al., 2013; Pun et al., 2018; Van den Berg et al., 2016; White et al., 2018; Y. Zhang et al., 2015; Zijlema et al., 2017).

Similar to search part 1, only two articles describe research on virtual reality representations of nature to improve well-being for older adults; the same articles identified in search part 1 (i.e., White et al. [2018] and Bruun-Pedersen et al. [2015a]).
To conclude, the aim of this search was to identify current insights in studies on benefits of virtual reality representations of nature on well-being of elderly. In the final search, we decided to redefine our search and exclude the search key words of Connectedness with the aim to identify more technology-related articles and repeat the database search.

\section{Search Part 3}

For this search, we used the search key words and synonyms for Technology, Nature, and Elderly. Other than that, the search strategy was equal to the previous searches.

\section{Results Part 3}

After checking for duplicates, the number of unique articles selected from the databases was 143 . The table of all hits that were not included in part 1 or part 2 is presented in Appendix 3.

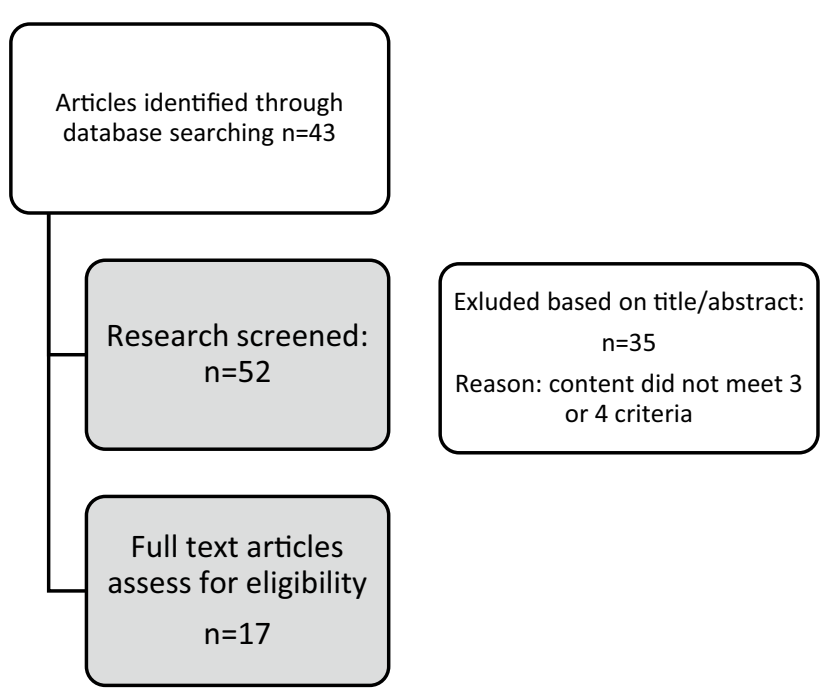

Fig. 2 Selection procedure Search Part 2 
Table 4 Articles identified in Search Part 2

\begin{tabular}{lll}
\hline Reference & Title & Main findings \\
\hline Akpinar et al. (2016) & $\begin{array}{c}\text { Does green space matter? Exploring relationships between } \\
\text { green space type and health indicators }\end{array}$ & $\begin{array}{c}\text { This study suggested that types of green space should be } \\
\text { considered individually rather than aggregated as "sim- } \\
\text { ply green." Additionally, "size" of forest in urban areas } \\
\text { seemed an important factor impacting the relationship } \\
\text { between green space and mental health. }\end{array}$ \\
\hline
\end{tabular}

\section{Study Characteristics}

Only articles that met all three criteria were included in the in-depth analysis. Two studies were identified, of which 1 (White et al., 2018) was already described in Table 2. Table 5 presents the remaining included study and its main findings.

Only three articles were identified that met all search criteria. Next, we will discuss these articles in more detail to generate understanding of the current knowledge base within the field of virtual reality research and digital nature representation.

In the article of Bruun-Pedersen et al. (2015b), a design approach for recreational virtual nature for elderly is proposed, with the possibility of implementation in rehabilitation health settings. This article is a follow-up on a pilot study in which nursing home residents were exposed to an augmented alternative for their daily biking exercise to improve physical well-being (Bruun-Pedersen et al., 2014). In this study, the authors proposed a set of guidelines with design considerations such as navigation guidelines and guidelines for content types of potential nature landmarks which might be used in recreational virtual environments. The authors conclude that the guidelines are based on literature and need further testing in real life settings.

The second article by White et al. (2018) is a review article on the possible uses of virtual nature in therapeutics to improve quality of life. The authors argue that when real interaction with nature is not possible or feasible, for example, for elderly with mobility issues, virtual nature could be considered as an alternative. The authors mention that there are several implementation possibilities of virtual nature or virtual reality in general in health environments. White et al. further recommend to also keep in mind the risks, benefits, and cost efficiency of these implementations but do not further describe them in much detail in their article.
The last study identified by our review that met all criteria is the study by Battisto et al. (2018). In this article, the authors discuss technological possibilities to increase nature interaction for older adults. They argue that technology could be used to make therapeutic landscapes accessible for older adults to promote health and to improve quality of life (Battisto et al., 2018). Subsequently, several examples of implementations are discussed, such as simulations, virtual nature environments, and interactive displays. According to Battisto et al. (2018), more research is needed in the field, and advanced technological solutions should be developed, especially for the design of convincing and realistic settings that provide the user with a feeling of actually being present in the virtual environment.

In conclusion, the three studies identified generate preliminary evidence for the effectiveness of virtual (nature) environments as a means to improve well-being amongst older adults. These studies provide starting points for the design and (technological) development of such environments. However, as of yet, there is no evidencebased design approach that generates understanding of how specific characteristics of virtual nature environments impact social well-being measures in particular, and of the underlying psychological processes involved.

\section{Discussion}

In the present paper, a rapid literature review consisting of three parts was reported with the aim to identify articles that focus on the effects of nature interaction on (social) wellbeing for older adults, and specifically, articles focussing on virtual representations of nature for older adults as a means to improve social well-being. In total, 29 unique articles were identified across the three searches that met at least

Table 5 Articles identified in search part 3

\begin{tabular}{lll}
\hline Reference & Title & Main findings \\
\hline Battisto et al. (2018) & $\begin{array}{c}\text { Technological supports to increase nature contact for older } \\
\text { adults }\end{array}$ & $\begin{array}{c}\text { This study stressed the opportunities to utilize technolo- } \\
\text { gies for connecting older adults to nature and described } \\
\text { challenges related to the creation of immersive, high } \\
\text { fidelity, realistic nature settings which could function as } \\
\text { a substitute for contact with real nature. }\end{array}$ \\
\hline
\end{tabular}


three of the four criteria (aim 1). Of these 29 articles, only three articles were identified using virtual representations of nature for elderly focussing on promoting general health (Battisto et al., 2018), recreation, and rehabilitation (BruunPedersen et al., 2015b) and quality of life (White et al., 2018). None of these articles, however, specifically aimed at improving social well-being of elderly users.

As such, the searches reported on in the present undertaking clearly point at a blind spot in contemporary research. Whereas there is a considerable body of research when zooming in on the research topics in isolation, there is very little cross-disciplinary research combining these topics by connecting insights from the social sciences with technology research and development. This connection is essential for successful implementation of virtual representations of nature in the lives of older adults.

Articles identified in this review mostly focus on the effects of (nearby) green space and mental health, such as reducing stress or improving quality of life. This body of research underscores the importance of (amongst others) available, nearby or urban green space for the mental health of the (ageing) population. These studies, however, do not contribute to solutions or innovations that make nature accessible for those with limited access to nature. Although many studies were identified using GPS or GIS data for data collection, only an extremely small number of studies using other types of technologies were identified. When considering the many ways in which digital nature could be presented to older adults using diverse technologies (such as virtual or augmented reality, smart screens, interactive walls, smart projections and so on), research exploring and testing effects (also taking into account frequency and duration of exposure) is highly called for.

Clearly, future research is warranted to unravel which digital types of nature could improve well-being for older adults, and to what extent such interventions can remedy social wellbeing (including loneliness and feelings of connectedness) in particular. In terms of urgency, bringing nature inside would be especially beneficial to older adults with mobility issues and to those living in urbanized regions where nature is scarce (Battisto et al., 2018; Browning et al., 2020; White et al., 2018).

Finally, the present review (including the three studies identified in the final search iteration) did not yield insights as to what specific virtual representations of nature characteristics are associated with improvements in (social) well-being. We aimed to identify studies reporting on preferences of older adults not only in real life nature (cf. Wen et al., 2018), but especially within virtual nature environments. According to a review article (Depledge et al., 2011), landscape features tested most frequently within virtual environments are concrete elements such as trees, people, and water. However, their effects on social well-being in particular were not tested. Additionally, these studies do not aim at identifying how more abstract visual-spatial characteristics such as spatial configuration, spaciousness, and perceived enclosure in digital nature environments impact outcome measures. Although specific visual-spatial features in augmented nature scenes like spaciousness have been shown to influence social aspirations within a student population (van Houwelingen-Snippe et al., 2020a, b), research is needed to identify whether such characteristics can also enhance (social) well-being and related measures amongst older adults.

Specifically related to the present pandemic (COVID19), social and mental well-being problems are predicted to aggravate in the upcoming period (Simon et al., 2020). These specific times bring many challenges with them for everyone, but especially so for older adults who are generally more vulnerable and for whom going outside might be even more of a challenge. When also considering the many restrictions worldwide, the importance of virtual representations of nature for older adults cannot be overstated.

\section{Limitations}

The number of studies matching all criteria was extremely limited. Table 2 indicates that although the total number of papers found with the isolated topics was substantial, clearly this was not the case for papers combining multiple topics. Hence, our findings call for multidisciplinary research approaches integrating findings from the domains of gerontology, nature research, and human media interaction research. Considering the limited number of papers, we did not include additional criteria (e.g., type of study, strength of evidence) to control for quality and relevance of the selected papers.

Arguably, our search key words were rather broad (e.g., the search key words concerning Elderly), which may have resulted in a failure to identify papers targeted at very specific patient groups or papers targeting age-related health problems including dementia or Parkinson's disease. For these patient groups, however, digital nature is often used as a means of recovery from fatigue or for recreational purposes (e.g., visiting a tropical island as a welcome distraction from daily concerns), rather than as a means for improving social well-being.

\section{Conclusions}

This rapid review points at a lack of studies combining insights of geriatric studies, nature studies, and humansystem interaction studies. Considering the diverse benefits of contact with nature to an ageing population and the many possibilities smart technologies provide for bringing nature inside, this review shows that opportunities for challenging, boundary-spanning research approaches to one of the most pressing societal challenges of our times are many. 


\section{Appendix 1}

Table 6 Table search part 1

\begin{tabular}{|c|c|c|c|c|}
\hline & $\begin{array}{l}\text { Nature inter- } \\
\text { action }\end{array}$ & Technology & Well-being & $\begin{array}{l}\text { Older adults } \\
\text { (not excluded) }\end{array}$ \\
\hline Astell-Burt et al. (2013) & $\mathrm{x}$ & & $\mathrm{x}$ & $\mathrm{x}$ \\
\hline Astell-Burt et al. (2016) & $\mathrm{x}$ & & $\mathrm{x}$ & $\mathrm{x}$ \\
\hline Bell et al. (2015) & $\mathrm{x}$ & GPS & $\mathrm{x}$ & $\mathrm{x}$ \\
\hline Bentsen et al. (2009) & $\mathrm{x}$ & & $\mathrm{x}$ & \\
\hline Bentsen et al. (2010) & $\mathrm{x}$ & & & \\
\hline Bernatzky (1975) & $\mathrm{x}$ & & $\mathrm{x}$ & \\
\hline Bodin et al. (2015) & & GPS & $\mathrm{x}$ & $\mathrm{x}$ \\
\hline Boeyen et al. (2017) & $\mathrm{x}$ & & & \\
\hline Bos et al. (2016) & $\mathrm{x}$ & & $\mathrm{x}$ & $\mathrm{x}$ \\
\hline Botticello et al. (2015) & & GPS & & $\mathrm{x}$ \\
\hline Bruun-Pedersen et al. (2015a) & $\mathrm{x}$ & $\mathrm{x}$ & $\mathrm{x}$ & $\mathrm{x}$ \\
\hline Büssing et al. (2005) & & & $\mathrm{x}$ & $\mathrm{x}$ \\
\hline Cartwright et al. (2018) & $\mathrm{x}$ & & $\mathrm{x}$ & \\
\hline Claessens et al. (2014) & $\mathrm{x}$ & GPS & & \\
\hline Costello et al. (2019) & $\mathrm{x}$ & & $\mathrm{x}$ & $\mathrm{x}$ \\
\hline Coutts et al. (2013) & $\mathrm{x}$ & & & $\mathrm{x}$ \\
\hline Dempsey et al. (2018a) & $\mathrm{x}$ & & $\mathrm{x}$ & $\mathrm{x}$ \\
\hline Dzhambov et al. (2018) & $\mathrm{x}$ & GPS & & \\
\hline \multicolumn{5}{|l|}{ Eckenwiler (2018) } \\
\hline Egorov et al. (2017) & $\mathrm{x}$ & GPS & $\mathrm{x}$ & $\mathrm{x}$ \\
\hline Engemann et al. (2019) & $\mathrm{x}$ & GPS & $\mathrm{x}$ & \\
\hline Ferrara et al. (2019) & $\mathrm{x}$ & GPS & & $\mathrm{x}$ \\
\hline Foster et al. (2009) & $\mathrm{x}$ & GPS & & $\mathrm{x}$ \\
\hline \multicolumn{5}{|l|}{ de Gelder et al. (2017) } \\
\hline Generaal et al. (2019) & & GPS & $\mathrm{x}$ & \\
\hline \multicolumn{5}{|l|}{ Germenis (2014) } \\
\hline Goyder et al. (2014) & & GPS & & $\mathrm{x}$ \\
\hline Grazuleviciene et al. (2016) & $\mathrm{x}$ & & & $\mathrm{x}$ \\
\hline Grigsby-Toussaint et al. (2015) & $\mathrm{x}$ & & $\mathrm{x}$ & $\mathrm{x}$ \\
\hline Guite et al. (2006) & $\mathrm{x}$ & & $\mathrm{x}$ & $\mathrm{x}$ \\
\hline Helbich (2019) & $\mathrm{x}$ & GPS & $\mathrm{x}$ & $\mathrm{x}$ \\
\hline Helbich et al. (2018) & $\mathrm{x}$ & GPS & $\mathrm{x}$ & $\mathrm{x}$ \\
\hline Helbich et al. (2016) & $\mathrm{x}$ & GPS & & \\
\hline Huang et al. (2020) & $\mathrm{x}$ & & & $\mathrm{x}$ \\
\hline Hunter et al. (2019) & $\mathrm{x}$ & & $\mathrm{x}$ & $\mathrm{x}$ \\
\hline Huynh et al. (2013) & $\mathrm{x}$ & GPS & $\mathrm{x}$ & \\
\hline Jansen et al. (2017) & $\mathrm{x}$ & GPS & & $\mathrm{x}$ \\
\hline Kessel et al. (2009) & $\mathrm{x}$ & GPS & & \\
\hline Kondo et al. (2017) & $\mathrm{x}$ & GPS & & \\
\hline Kondo et al. (2020) & $\mathrm{x}$ & GPS & $\mathrm{x}$ & $\mathrm{x}$ \\
\hline \multicolumn{5}{|l|}{ Kumagai et al. (2015) } \\
\hline Lanki et al. (2017) & $\mathrm{x}$ & & & \\
\hline Lee and Lee (2019) & $\mathrm{x}$ & & $\mathrm{x}$ & $\mathrm{x}$ \\
\hline Li and Ghosh (2018) & $\mathrm{x}$ & Google Street View & & $\mathrm{x}$ \\
\hline Liang et al. (2017) & $\mathrm{x}$ & GPS & & \\
\hline Liao et al. (2019) & $\mathrm{x}$ & & & \\
\hline Liao et al. (2019a, b) & $\mathrm{x}$ & & & \\
\hline \multicolumn{5}{|l|}{ Liddicoat et al. (2020) } \\
\hline Lin et al. (2018) & $\mathrm{x}$ & GPS & & \\
\hline
\end{tabular}


Table 6 (continued)

\begin{tabular}{|c|c|c|c|c|}
\hline & $\begin{array}{l}\text { Nature inter- } \\
\text { action }\end{array}$ & Technology & Well-being & $\begin{array}{l}\text { Older adults } \\
\text { (not excluded) }\end{array}$ \\
\hline Logan et al. (2015) & $\mathrm{x}$ & & $\mathrm{x}$ & \\
\hline Lyu et al. (2018) & $\mathrm{x}$ & & $\mathrm{x}$ & \\
\hline Ma et al. (2018) & $\mathrm{x}$ & GPS & & $\mathrm{x}$ \\
\hline Magalhães et al. (2017) & $\mathrm{x}$ & & & \\
\hline Mäki-Opas et al. (2016) & $\mathrm{x}$ & GPS & & $\mathrm{x}$ \\
\hline \multicolumn{5}{|l|}{ Manferdelli et al. (2019) } \\
\hline McGrath et al. (2015) & $\mathrm{x}$ & GPS & & \\
\hline Miralles-Guasch et al. (2019) & $\mathrm{x}$ & GPS & & $\mathrm{x}$ \\
\hline Mukherjee et al. (2017b) & $\mathrm{x}$ & GPS & $\mathrm{x}$ & $\mathrm{x}$ \\
\hline Müller et al. (2018) & $\mathrm{x}$ & GPS & & $\mathrm{x}$ \\
\hline Mygind et al. (2018) & $\mathrm{x}$ & & $\mathrm{x}$ & \\
\hline Nakau et al. (2013) & $\mathrm{x}$ & & $\mathrm{x}$ & $\mathrm{x}$ \\
\hline Nichani et al. (2017) & $\mathrm{x}$ & GPS & $\mathrm{x}$ & \\
\hline Noordzij et al. (2020) & $\mathrm{x}$ & & $\mathrm{x}$ & $\mathrm{x}$ \\
\hline Nordbø et al. (2019) & $\mathrm{x}$ & GPS & $\mathrm{x}$ & \\
\hline Nutsford et al. (2013) & $\mathrm{x}$ & & $\mathrm{x}$ & $\mathrm{x}$ \\
\hline Ord et al. (2017) & $\mathrm{x}$ & & & $\mathrm{x}$ \\
\hline Paquet et al. (2013) & $\mathrm{x}$ & & & $\mathrm{x}$ \\
\hline Park (2017) & $\mathrm{x}$ & GPS & & \\
\hline Patel et al. (2019) & $\mathrm{x}$ & GPS & $\mathrm{x}$ & $\mathrm{x}$ \\
\hline Pereira et al. (2019) & $\mathrm{x}$ & & & \\
\hline Puhakka et al. (2018) & $\mathrm{x}$ & GPS & & \\
\hline Pun et al. (2018) & $\mathrm{x}$ & & $\mathrm{x}$ & $\mathrm{x}$ \\
\hline Rahman and Zhang (2018) & $\mathrm{x}$ & Google Earth & & $\mathrm{x}$ \\
\hline Reid et al. (2009) & & & & $\mathrm{x}$ \\
\hline Ribeiro et al. (2019) & $\mathrm{x}$ & & $\mathrm{x}$ & \\
\hline Rook (2013) & $\mathrm{x}$ & & $\mathrm{x}$ & \\
\hline Rook et al. (2014) & $\mathrm{x}$ & & $\mathrm{x}$ & \\
\hline \multicolumn{5}{|l|}{ Servadio et al. (2019) } \\
\hline Stewart et al. (2018a) & $\mathrm{x}$ & GPS & & $\mathrm{x}$ \\
\hline Storgaard et al. (2013) & $\mathrm{x}$ & GPS & & $\mathrm{x}$ \\
\hline Su et al. (2019) & $\mathrm{x}$ & GPS & $\mathrm{x}$ & \\
\hline Sugiyama et al. (2016) & $\mathrm{x}$ & & $\mathrm{x}$ & \\
\hline Sun et al. (2017) & & GPS & & \\
\hline Tan et al. (2007) & & & & $\mathrm{x}$ \\
\hline Triguero-Mas et al. (2017) & $\mathrm{x}$ & & $\mathrm{x}$ & $\mathrm{x}$ \\
\hline van den Bosch et al. (2015) & $\mathrm{x}$ & GPS & $\mathrm{x}$ & $\mathrm{x}$ \\
\hline Vienneau et al. (2017) & $\mathrm{x}$ & & & \\
\hline Vogt et al. (2015) & $\mathrm{x}$ & GPS & $\mathrm{x}$ & $\mathrm{x}$ \\
\hline Wang et al. $(2017 \mathrm{a}, \mathrm{b})$ & $\mathrm{x}$ & & & $\mathrm{x}$ \\
\hline Wang et al. (2019) & $\mathrm{x}$ & GPS & & $\mathrm{x}$ \\
\hline Wang et al. $(2019 a, b)$ & & & & $\mathrm{x}$ \\
\hline White et al. (2018) & $\mathrm{x}$ & $\mathrm{x}$ & $\mathrm{x}$ & $\mathrm{x}$ \\
\hline \multicolumn{5}{|l|}{ Younan et al. (2018) } \\
\hline Zandieh et al. (2019) & $\mathrm{x}$ & GPS & & $\mathrm{x}$ \\
\hline Zhang et al. (2015) & $\mathrm{x}$ & & $\mathrm{x}$ & $\mathrm{x}$ \\
\hline Zhang et al. (2019) & $\mathrm{x}$ & GPS & $\mathrm{x}$ & $\mathrm{x}$ \\
\hline Zijlema et al. (2017) & $\mathrm{x}$ & & $\mathrm{x}$ & $\mathrm{x}$ \\
\hline
\end{tabular}




\section{Appendix 2}

Table 7 Table search part 2 (excluding articles selected in part 1)

\begin{tabular}{|c|c|c|c|c|}
\hline & Nature & Technology & Connectedness & $\begin{array}{l}\text { Older } \\
\text { adults (not } \\
\text { excluded) }\end{array}$ \\
\hline Akpinar et al. (2016) & $\mathrm{x}$ & & $\mathrm{x}$ & $\mathrm{x}$ \\
\hline Ashbullby et al. (2013) & $\mathrm{x}$ & & $\mathrm{x}$ & \\
\hline \multicolumn{5}{|l|}{ Debele (2014) } \\
\hline Moffat et al. (2009) & $\mathrm{x}$ & & & \\
\hline Villeneuve et al. (2012) & $\mathrm{x}$ & & & \\
\hline Younan et al. (2016) & $\mathrm{x}$ & & $\mathrm{x}$ & \\
\hline
\end{tabular}

\section{Appendix 3}

Table 8 Table search part 3 (excluding articles selected in part 1 or 2)

\begin{tabular}{|c|c|c|c|}
\hline & Nature & Technology & $\begin{array}{l}\text { Older } \\
\text { adults (not } \\
\text { excluded) }\end{array}$ \\
\hline Almeter et al. (2018) & $\mathrm{x}$ & GPS & \\
\hline Astell-Burt et al. (2016) & $\mathrm{x}$ & GPS & $\mathrm{x}$ \\
\hline Barbosa et al. (2007) & $\mathrm{x}$ & GPS & $\mathrm{x}$ \\
\hline Battisto et al. (2018) & $\mathrm{x}$ & $\mathrm{x}$ & $\mathrm{x}$ \\
\hline Benmarhnia et al. (2017) & & & $\mathrm{x}$ \\
\hline Bunney et al. (2016) & $\mathrm{x}$ & GPS & \\
\hline Burgoine et al. (2015) & $\mathrm{x}$ & GPS & \\
\hline Cassarino et al. (2019) & $\mathrm{x}$ & $\mathrm{x}$ & \\
\hline Chien et al. (2019) & & GPS & $\mathrm{x}$ \\
\hline Cochrane et al. (2009) & $\mathrm{x}$ & GPS & $\mathrm{x}$ \\
\hline Coutts et al. (2010) & $\mathrm{x}$ & GPS & $\mathrm{x}$ \\
\hline Cui et al. (2013) & $\mathrm{x}$ & & \\
\hline Cumo et al. (2017) & $\mathrm{x}$ & & $\mathrm{x}$ \\
\hline Dadvand et al. (2017) & $\mathrm{x}$ & & \\
\hline Douglas et al. (2018) & $\mathrm{x}$ & GPS & $\mathrm{x}$ \\
\hline Forsyth and Crewe (2010) & $\mathrm{x}$ & & \\
\hline Gao et al. (2012) & $\mathrm{x}$ & & \\
\hline Germann-Chiari and Seeland (2004) & $\mathrm{x}$ & GPS & $\mathrm{x}$ \\
\hline Gose et al. (2013) & & GPS & \\
\hline Graça et al. (2018) & $\mathrm{x}$ & & $\mathrm{x}$ \\
\hline Green et al. (2016) & $\mathrm{x}$ & & \\
\hline Haggag (2010) & $\mathrm{x}$ & & \\
\hline Hermida et al. (2017) & $\mathrm{x}$ & GPS & $\mathrm{x}$ \\
\hline Hillsdon et al. (2006) & $\mathrm{x}$ & GPS & $\mathrm{x}$ \\
\hline Hoffimann et al. (2017) & $\mathrm{x}$ & GPS & \\
\hline Huang et al. (2018) & $\mathrm{x}$ & & $\mathrm{x}$ \\
\hline Hui et al. (2017) & $\mathrm{x}$ & & \\
\hline Jamaludin et al. (2014) & $\mathrm{x}$ & & \\
\hline Janssen and Rosu (2015) & $\mathrm{x}$ & GPS & \\
\hline Jim and Chen (2007) & $\mathrm{x}$ & & \\
\hline Jim and Shan (2013) & $\mathrm{x}$ & & $\mathrm{x}$ \\
\hline Jones (2018) & $\mathrm{x}$ & & \\
\hline King et al. (2015) & $\mathrm{x}$ & & $\mathrm{x}$ \\
\hline King et al. (2012) & $\mathrm{x}$ & GPS & $\mathrm{x}$ \\
\hline
\end{tabular}


Table 8 (continued)

\begin{tabular}{|c|c|c|c|}
\hline & Nature & Technology & $\begin{array}{l}\text { Older } \\
\text { adults (not } \\
\text { excluded) }\end{array}$ \\
\hline \multicolumn{4}{|l|}{$\overline{\text { Kruuse Afverchou (2005) }}$} \\
\hline Lachowycz et al. (2012) & $\mathrm{x}$ & GPS & \\
\hline Li and Ghosh (2018) & $\mathrm{x}$ & Google Street View & $\mathrm{x}$ \\
\hline \multicolumn{4}{|l|}{ Li et al. (2008) } \\
\hline Lin et al. (2018) & $\mathrm{x}$ & GPS & \\
\hline Liu et al. (2017) & $\mathrm{x}$ & & $\mathrm{x}$ \\
\hline Marquet et al. (2019) & $\mathrm{x}$ & GPS & \\
\hline Michimi and Wimberly (2012) & $\mathrm{x}$ & GPS & $\mathrm{x}$ \\
\hline \multicolumn{4}{|l|}{ Mihrshahi et al. (2018) } \\
\hline Møller et al. (2019) & $\mathrm{x}$ & GPS & \\
\hline \multicolumn{4}{|l|}{ Morris et al. (2006) } \\
\hline Ngom et al. (2016) & $\mathrm{x}$ & GPS & $\mathrm{x}$ \\
\hline Occhiuto (2018) & $\mathrm{x}$ & & \\
\hline Othman et al. (2015) & $\mathrm{x}$ & $\mathrm{x}$ & \\
\hline Panyadee et al. (2016) & $\mathrm{x}$ & & $\mathrm{x}$ \\
\hline Potestio et al. (2009) & $\mathrm{x}$ & GPS & \\
\hline Pourzargar (2016) & $\mathrm{x}$ & & \\
\hline Prince et al. (2011) & $\mathrm{x}$ & & $\mathrm{x}$ \\
\hline Rahman et al. (2019) & $\mathrm{x}$ & GPS & \\
\hline Rahman and Zhang (2018) & $\mathrm{x}$ & GPS & $\mathrm{x}$ \\
\hline Raymond et al. (2016) & $\mathrm{x}$ & GPS & $\mathrm{x}$ \\
\hline Ribeiro et al. (2015) & $\mathrm{x}$ & GPS & $\mathrm{x}$ \\
\hline Richardson et al. (2017) & $\mathrm{x}$ & GPS & $\mathrm{x}$ \\
\hline Rudnev (2012) & $\mathrm{x}$ & & \\
\hline Saghafi and Ahmadpour (2017) & $\mathrm{x}$ & & $\mathrm{x}$ \\
\hline Sanchez et al. (2010) & $\mathrm{x}$ & & $\mathrm{x}$ \\
\hline Shackleton et al. (2015) & $\mathrm{x}$ & & $\mathrm{x}$ \\
\hline \multicolumn{4}{|l|}{ Shourbela et al. (2016) } \\
\hline Shrestha et al. (2018) & $\mathrm{x}$ & & \\
\hline Son et al. (2019) & $\mathrm{x}$ & & $\mathrm{x}$ \\
\hline Stewart et al. (2018b) & $\mathrm{x}$ & GPS & $\mathrm{x}$ \\
\hline Sugiyama et al. (2014) & $\mathrm{x}$ & GPS & $\mathrm{x}$ \\
\hline Sun et al. (2017) & $\mathrm{x}$ & GPS & $\mathrm{x}$ \\
\hline Tan et al. (2007) & $\mathrm{x}$ & & $\mathrm{x}$ \\
\hline Tian and Jim (2012) & $\mathrm{x}$ & & \\
\hline Tian et al. (2014) & $\mathrm{x}$ & & \\
\hline Tikka et al. (2000) & $\mathrm{x}$ & & \\
\hline Uitto et al. (2006) & $\mathrm{x}$ & & \\
\hline Veitch et al. (2015) & $\mathrm{x}$ & GPS & $\mathrm{x}$ \\
\hline Wang et al. $(2017 \mathrm{a}, \mathrm{b})$ & $\mathrm{x}$ & & $\mathrm{x}$ \\
\hline Wang et al. (2016) & $\mathrm{x}$ & GPS & $\mathrm{x}$ \\
\hline Wang et al. $(2019 a, b)$ & $\mathrm{x}$ & GPS & $\mathrm{x}$ \\
\hline \multicolumn{4}{|l|}{ Wang et al. (2017) } \\
\hline Wang and Liu (2017) & $\mathrm{x}$ & & \\
\hline Xie et al. (2018) & $\mathrm{x}$ & & \\
\hline Xu and Gao (2017) & $\mathrm{x}$ & & $\mathrm{x}$ \\
\hline Xu et al. (2019) & $\mathrm{x}$ & & \\
\hline \multicolumn{4}{|l|}{ Zacharias et al. (2015) } \\
\hline Zhu and Jia (2012) & $\mathrm{x}$ & & \\
\hline
\end{tabular}


Acknowledgements The authors would like to thank Kars Otten for his work as second reviewer.

Funding The authors disclosed receipt of the following financial support for the research, authorship, and/or publication of this article: This research was funded by a grant from the Dutch Research Council (NWO).

\section{Declarations}

Conflict of Interest The authors declare that they have no conflict of interest

Open Access This article is licensed under a Creative Commons Attribution 4.0 International License, which permits use, sharing, adaptation, distribution and reproduction in any medium or format, as long as you give appropriate credit to the original author(s) and the source, provide a link to the Creative Commons licence, and indicate if changes were made. The images or other third party material in this article are included in the article's Creative Commons licence, unless indicated otherwise in a credit line to the material. If material is not included in the article's Creative Commons licence and your intended use is not permitted by statutory regulation or exceeds the permitted use, you will need to obtain permission directly from the copyright holder. To view a copy of this licence, visit http://creativecommons.org/licenses/by/4.0/.

\section{References}

Akpinar, A., Barbosa-Leiker, C., \& Brooks, K. R. (2016). Does green space matter? Exploring relationships between green space type and health indicators. Urban Forestry and Urban Greening, 20, 407-418. https://doi.org/10.1016/j.ufug.2016.10.013.

Almeter, A., Tashie, A., Procter, A., McAlexander, T., Browning, D., Rudder, C., \& Araujo, R. (2018). A needs-driven, multi-objective approach to allocate urban ecosystem services from 10,000 Trees. Sustainability (Switzerland), 10(12). https://doi. org/10.3390/su10124488.

Annerstedt, M., Jönsson, P., Wallergård, M., Johansson, G., Karlson, B., Grahn, P., \& Währborg, P. (2013). Inducing physiological stress recovery with sounds of nature in a virtual reality forestresults from a pilot study. Physiology and Behavior, 118, 240250. https://doi.org/10.1016/j.physbeh.2013.05.023.

Annerstedt, M., \& Währborg, P. (2011). Nature-assisted therapy: Systematic review of controlled and observational studies. Scandinavian Journal of Public Health, 39(4), 371-388. https:// doi.org/10.1177/1403494810396400.

Ashbullby, K. J., Pahl, S., Webley, P., \& White, M. P. (2013). The beach as a setting for families' health promotion: A qualitative study with parents and children living in coastal regions in Southwest England. Health and Place, 23, 138-147. https://doi. org/10.1016/j.healthplace.2013.06.005.

Astell-Burt, T., Feng, X., \& Kolt, G. S. (2013). Mental health benefits of neighbourhood green space are stronger among physically active adults in middle-to-older age: Evidence from 260,061 Australians. Preventive Medicine, 57(5), 601-606. https://doi. org/10.1016/j.ypmed.2013.08.017.

Astell-Burt, T., Feng, X., \& Kolt, G. S. (2016). Large-scale investment in green space as an intervention for physical activity, mental and cardiometabolic health: Study protocol for a quasi-experimental evaluation of a natural experiment. BMJ Open, 6(4). https://doi. org/10.1136/bmjopen-2015-009803.
Baños, R. M., Etchemendy, E., Castilla, D., García-Palacios, A., Quero, S., \& Botella, C. (2012). Positive mood induction procedures for virtual environments designed for elderly people. Interacting with Computers. https://doi.org/10.1016/j.intcom.2012.04.002.

Barbosa, O., Tratalos, J. A., Armsworth, P. R., Davies, R. G., Fuller, R. A., Johnson, P., \& Gaston, K. J. (2007). Who benefits from access to green space? A case study from Sheffield UK. Landscape and Urban Planning, 83(2-3), 187-195. https://doi.org/10.1016/j. landurbplan.2007.04.004.

Battisto, D., Vincent, E., \& Dye, C. J. (2018). Technological supports to increase nature contact for older adults. Agint, Technology and Health. https://doi.org/10.1016/B978-0-12-811272-4.00005-1.

Bell, S. L., Phoenix, C., Lovell, R., \& Wheeler, B. W. (2015). Seeking everyday wellbeing: The coast as a therapeutic landscape. Social Science and Medicine, 142, 56-67. https://doi.org/10.1016/j. socscimed.2015.08.011.

Benmarhnia, T., Kihal-Talantikite, W., Ragettli, M. S., \& Deguen, S. (2017). Small-area spatiotemporal analysis of heatwave impacts on elderly mortality in Paris: A cluster analysis approach. Science of the Total Environment, 592, 288-294. https://doi. org/10.1016/j.scitotenv.2017.03.102.

Bentsen, P., Mygind, E., \& Randrup, T. B. (2009). Towards an understanding of udeskole: Education outside the classroom in a danish context. Education 3-13, 37(1), 29-44. https://doi.org/10.1080/ 03004270802291780.

Bentsen, P., Søndergaard Jensen, F., Mygind, E., \& Barfoed Randrup, T. (2010). The extent and dissemination of udeskole in Danish schools. Urban Forestry and Urban Greening, 9(3), 235-243. https://doi.org/10.1016/j.ufug.2010.02.001.

Bernatzky, A. (1975). Gardens for stepped terrace housing. Urban Ecology, 1(1), 49-62. https://doi.org/10.1016/03044009(75)90004-2.

Bodin, T., Björk, J., Ardö, J., \& Albin, M. (2015). Annoyance, sleep and concentration problems due to combined traffic noise and the benefit of quiet Side. International Journal of Environmental Research and Public Health, 12(2), 1612-1628. https://doi. org/10.3390/ijerph120201612.

Boeyen, J., Callan, A. C., Blake, D., Wheeler, A. J., Franklin, P., Hall, G. L., \& Hinwood, A. (2017). Investigating the relationship between environmental factors and respiratory health outcomes in school children using the forced oscillation technique. International Journal of Hygiene and Environmental Health, 220(2), 494-502. https://doi.org/10.1016/j.ijheh.2017.01.014.

Bos, E. H., van der Meulen, L., Wichers, M., \& Jeronimus, B. F. (2016). A primrose path? Moderating effects of age and gender in the association between green space and mental health. International Journal of Environmental Research and Public Health, 13(5). https://doi.org/10.3390/ijerph13050492.

Botticello, A. L., Rohrbach, T., \& Cobbold, N. (2015). Differences in the community built environment influence poor perceived health among persons with spinal cord injury. Archives of Physical Medicine and Rehabilitation, 96(9), 1583-1590. https://doi. org/10.1016/j.apmr.2015.04.025.

Bratman, G. N., Anderson, C. B., Berman, M. G., Cochran, B., de Vries, S., Flanders, J., \& Daily, G. C. (2019). Nature and mental health: An ecosystem service perspective. Science Advances, 5(7). https:// doi.org/10.1126/sciadv.aax0903.

Browning, M. H. E. M., Mimnaugh, K. J., van Riper, C. J., Laurent, H. K., \& LaValle, S. M. (2020). Can simulated nature support mental health? Comparing short, single-doses of 360-degree nature videos in virtual reality with the outdoors. Frontiers in Psychology, 10(January), 1-14. https://doi.org/10.3389/fpsyg. 2019.02667.

Bruun-Pedersen, J. R., Pedersen, K. S., Serafin, S., \& Kofoed, L. B. (2014). Augmented exercise biking with virtual environments for elderly users: A preliminary study for retirement home 
physical therapy. 2014 2nd Workshop on Virtual and Augmented Assistive Technology (VAAT), 23-27. https://doi.org/10.1109/ VAAT.2014.6799464.

Bruun-Pedersen, J. R., Serafin, S., \& Kofoed, L. B. (2016). Going outside while staying inside-exercise motivation with immersive vs. non-immersive recreational virtual environment augmentation for older adult nursing home residents. Proceedings - 2016 IEEE International Conference on Healthcare Informatics, ICHI 2016, 216-226. https://doi.org/10.1109/ICHI.2016.31.

Bruun-Pedersen, J. R., Stefania, S., \& Kofoed, L. B. (2015a). Simulating nature for elderly users - a design approach for recreational virtual environments. Proceedings - 15th IEEE International Conference on Computer and Information Technology, CIT 2015, 14th IEEE International Conference on Ubiquitous Computing and Communications, IUCC 2015, 13th IEEE International Conference on Dependable, Autonomic and Se, 1566-1571. https://doi. org/10.1109/CIT/IUCC/DASC/PICOM.2015.235.

Bruun-Pedersen, J. R., Stefania, S., \& Kofoed, L. B. (2015b). Simulating nature for elderly users-a design approach for recreational virtual environments. Proceedings - 15th IEEE International Conference on Computer and Information Technology, CIT 2015, 14th IEEE International Conference on Ubiquitous Computing and Communications, IUCC 2015, 13th IEEE International Conference on Dependable, Autonomic and Se, 8, 1566-1571. https://doi.org/10.1109/CIT/IUCC/DASC/PICOM.2015.235.

Bunney, P. E., Zink, A. N., Holm, A. A., Billington, C. J., \& Kotz, C. M. (2016). Impact of park renovations on park use and park-based physical activity. Physiology \& Behavior, 176(2), 139-148. https:// doi.org/10.1016/j.physbeh.2017.03.040.

Burgoine, T., Jones, A. P., Namenek Brouwer, R. J., \& Benjamin Neelon, S. E. (2015). Associations between BMI and home, school and route environmental exposures estimated using GPS and GIS: Do we see evidence of selective daily mobility bias in children? International Journal of Health Geographics, 14(1), 1-12. https://doi.org/10.1186/1476-072X-14-8.

Büssing, A., Matthiessen, P. F., \& Ostermann, T. (2005). Engagement of patients in religious and spiritual practices: Confirmatory results with the SpREUK-P 1.1 questionnaire as a tool of quality of life research. Health and Quality of Life Outcomes, 3, 1-11. https:// doi.org/10.1186/1477-7525-3-53.

Cacioppo, J. T., Chen, H. Y., \& Cacioppo, S. (2017). Reciprocal Influences between loneliness and self-centeredness: A crosslagged panel analysis in a population-based sample of African American, Hispanic, and Caucasian adults. Personality and Social Psychology Bulletin, 43(8), 1125-1135. https://doi. org/10.1177/0146167217705120.

Cartwright, B. D. S., White, M. P., \& Clitherow, T. J. (2018). Nearby nature 'buffers' the effect of low social connectedness on adult subjective wellbeing over the last 7 days. International Journal of Environmental Research and Public Health, 15(6). https://doi. org/10.3390/ijerph15061238.

Cassarino, M., Maisto, M., Esposito, Y., Guerrero, D., Chan, J. S., \& Setti, A. (2019). Testing attention restoration in a virtual reality driving simulator. Frontiers in Psychology, 10(FEB), 1-7. https:// doi.org/10.3389/fpsyg.2019.00250.

Chien, J.-W., Yang, Y.-R., Chen, S.-Y., Chang, Y.-J., \& Chan, C.-C. (2019). Urban open space is associated with better renal function of adult residents in New Taipei City. International Journal of Environmental Research and Public Health, 16(13), 2436. https:// doi.org/10.3390/ijerph16132436.

Claessens, J., Schram-Bijkerk, D., Dirven-van Breemen, L., Otte, P., \& van Wijnen, H. (2014). The soil-water system as basis for a climate proof and healthy urban environment: Opportunities identified in a Dutch case-study. Science of the Total Environment, 485-486(1), 776-784. https://doi.org/10.1016/j. scitotenv.2014.02.120.
Cochrane, T., Davey, R. C., Gidlow, C., Smith, G. R., Fairburn, J., Armitage, C. J., \& Speight, S. (2009). Small area and individual level predictors of physical activity in urban communities: A multi-level study in stoke on trent, England. International Journal of Environmental Research and Public Health, 6(2), 654-677. https://doi.org/10.3390/ijerph6020654.

Costello, L., McDermott, M. L., Patel, P., \& Dare, J. (2019). 'A lot better than medicine'-self-organised ocean swimming groups as facilitators for healthy ageing. Health and Place, 60 (October), 102212. https://doi.org/10.1016/j.healthplace.2019.102212.

Coutts, C., Chapin, T., Horner, M., \& Taylor, C. (2013). County-level effects of green space access on physical activity. Journal of Physical Activity and Health, 10(2).

Coutts, C., Horner, M., \& Chapin, T. (2010). Using geographical information system to model the effects of green space accessibility on mortality in Florida. Geocarto International, 25(6), 471-484. https://doi.org/10.1080/10106049.2010.505302.

Cui, Z., Wen, T., \& Zhang, Y. (2013). Research of space form evolution of new and high technology industries development zones. Advanced Materials Research. https://doi.org/10.4028/www. scientific.net/AMR.671-674.2431.

Cumo, F., Curreli, F. R., Pennacchia, E., Piras, G., \& Roversi, R. (2017). Enhancing the urban quality of life: A case study of a coastal city in the metropolitan area of Rome. WIT Transactions on the Built Environment, 170(October 2014), 127-137. https:// doi.org/10.2495/CC170131.

Dadvand, P., Tischer, C., Estarlich, M., Llop, S., Dalmau-Bueno, A., López-Vicente, M., \& Sunyer, J. (2017). Lifelong residential exposure to green space and attention: A population-based prospective study. Environmental Health Perspectives, 125(9), 1-8. https://doi.org/10.1289/EHP694.

De Gelder, R., Koster, E. M., van Buren, L. P., van Ameijden, E. J. C., Harrison, A., Birt, C. A., \& Verma, A. (2017). Differences in adults' health and health behaviour between 16 European urban areas and the associations with socio-economic status and physical and social environment. European Journal of Public Health, 27(2), 93-99. https://doi.org/10.1093/eurpub/ckv141.

De Koning, J. L., Stathi, A., \& Richards, S. (2017). Predictors of loneliness and different types of social isolation of rural-living older adults in the United Kingdom. Ageing and Society. https:// doi.org/10.1017/S0144686X16000696.

Debele, M. (2014). Utopianism and anti-utopianism in the ways older Ethiopian children construct their national identity and implications for social studies education, (c), 2-6.

Dempsey, S., Devine, M. T., Gillespie, T., Lyons, S., \& Nolan, A. (2018a). Coastal blue space and depression in older adults. Health and Place, 54(September), 110-117. https://doi. org/10.1016/j.healthplace.2018.09.002.

Dempsey, S., Devine, M. T., Gillespie, T., Lyons, S., \& Nolan, A. (2018b). Coastal blue space and depression in older adults. Health and Place, 54(June), 110-117. https://doi.org/10.1016/j. healthplace.2018.09.002.

Depledge, M. H., Stone, R. J., \& Bird, W. J. (2011). Can natural and virtual environments be used to promote improved human health and wellbeing? Environmental, Science \& Technology, 45, 46604665. https://doi.org/10.1021/es103907m.

Detweiler, M. B., Sharma, T., Detweiler, J. G., Murphy, P. F., Lane, S., Carman, J., \& Kim, K. Y. (2012). What is the evidence to support the use of therapeutic gardens for the elderly? Psychiatry Investigation, 9(2), 100-110. https://doi.org/10.4306/ pi.2012.9.2.100.

Dickens, A. P., Richards, S. H., Greaves, C. J., \& Campbell, J. L. (2011a). Interventions targeting social isolation in older people: A systematic review. BMC Public Health, 11(1), 647. https://doi. org/10.1186/1471-2458-11-647. 
Dickens, A. P., Richards, S. H., Greaves, C. J., \& Campbell, J. L. (2011b). Interventions targeting social isolation in older people: A systematic review. BMC Public Health. https://doi. org/10.1186/1471-2458-11-647.

Dodge, R., Daly, A., Huyton, J., \& Sanders, L. (2012). The challenge of defining wellbeing. International Journal of Wellbeing, 2(3), 222-235. https://doi.org/10.5502/ijw.v2i3.4.

Douglas, J. A., Briones, M. D., Bauer, E. Z., Trujillo, M., Lopez, M., \& Subica, A. M. (2018). Social and environmental determinants of physical activity in urban parks: Testing a neighborhood disorder model. Preventive Medicine, 109(January), 119-124. https://doi. org/10.1016/j.ypmed.2018.01.013.

Dzhambov, A. M., Markevych, I., Tilov, B., Arabadzhiev, Z., Stoyanov, D., Gatseva, P., \& Dimitrova, D. D. (2018). Lower noise annoyance associated with GIS-derived greenspace: Pathways through perceived greenspace and residential noise. International Journal of Environmental Research and Public Health, 15(7), 1-15. https://doi.org/10.3390/ijerph15071533.

Eckenwiler, L. (2018). Displacement and solidarity: An ethic of placemaking. Bioethics, 32(9), 562-568. https://doi.org/10.1111/ bioe. 12538 .

Egorov, A. I., Griffin, S. M., Converse, R. R., Styles, J. N., Sams, E. A., Wilson, A., \& Wade, T. J. (2017). Vegetated land cover near residence is associated with reduced allostatic load and improved biomarkers of neuroendocrine, metabolic and immune functions. Environmental Research, 158(July), 508-521. https:// doi.org/10.1016/j.envres.2017.07.009.

Engemann, K., Pedersen, C. B., Arge, L., Tsirogiannis, C., Mortensen, P. B., \& Svenning, J.-C. (2019). Residential green space in childhood is associated with lower risk of psychiatric disorders from adolescence into adulthood. Proceedings of the National Academy of Sciences, 116(11), 5188-5193. https://doi. org/10.1073/pnas.1807504116.

Fakoya, O. A., McCorry, N. K., \& Donnelly, M. (2020). Loneliness and social isolation interventions for older adults: A scoping review of reviews. BMC Public Health, 20(1), 129. https://doi. org/10.1186/s12889-020-8251-6.

Ferrara, E., Liotta, A., Ndubuaku, M., Erhan, L., Giusto, D., Richardson, M., \& McEwan, K. (2019). A demographic analysis of urban nature utilization. 2018 10th Computer Science and Electronic Engineering Conference, CEEC 2018 - Proceedings, 136-141. https://doi.org/10.1109/CEEC.2018.8674206.

Finlay, J., Franke, T., McKay, H., \& Sims-Gould, J. (2015). Therapeutic landscapes and wellbeing in later life: Impacts of blue and green spaces for older adults. Health and Place, 34, 97-106. https:// doi.org/10.1016/j.healthplace.2015.05.001.

Fiorini, R. A., De Giacomo, P., \& L'Abate, L. (2016). Wellbeing Understanding in High Quality Healthcare Informatics and Telepractice. Studies in health technology and informatics, 226, 153-156.

Forsyth, A., \& Crewe, K. (2010). Suburban technopoles as places: The international campus-garden-suburb style. Urban Design International. https://doi.org/10.1057/udi.2010.15.

Foster, C., Hillsdon, M., Jones, A., Grundy, C., Wilkinson, P., White, M., \& Thorogood, M. (2009). Objective measures of the environment and physical activity-results of the environment and physical activity study in english adults. Journal of Physical Activity and Health, 6(s1), S70-S80. https://doi.org/10.1123/jpah.6.s1.s70.

Ganann, R., Ciliska, D., \& Thomas, H. (2010). Expediting systematic reviews: Methods and implications of rapid reviews. Implementation Science, 5, 56.

Gao, T., Qiu, L., Hammer, M., \& Gunnarsson, A. (2012). The importance of temporal and spatial vegetation structure information in biotope mapping schemes: A case study in Helsingborg, Sweden. Environmental Management, 49(2), 459-472. https://doi.org/10.1007/s00267-011-9795-0.
Garritty, C., Stevens, A., Gartlehner, G., King, V., \& Kamel, C. (2016). Cochrane Rapid Reviews Methods Group to play a leading role in guiding the production of informed high-quality, timely research evidence syntheses. Systematic Reviews, 5, 184. https://doi. org/10.1186/s13643-016-0360-z.

Gascon, M., Mas, M. T., Martínez, D., Dadvand, P., Forns, J., Plasència, A., \& Nieuwenhuijsen, M. J. (2015). Mental health benefits of long-term exposure to residential green and blue spaces: A systematic review. International Journal of Environmental Research and Public Health, 12(4), 4354-4379. https://doi. org/10.3390/ijerph120404354.

Generaal, E., Hoogendijk, E. O., Stam, M., Henke, C. E., Rutters, F., Oosterman, M., \& Penninx, B. W. J. H. (2019). Neighbourhood characteristics and prevalence and severity of depression: Pooled analysis of eight Dutch cohort studies. British Journal of Psychiatry, 215(2), 468-475. https://doi.org/10.1192/ bjp.2019.100.

Germann-Chiari, C., \& Seeland, K. (2004). Are urban green spaces optimally distributed to act as places for social integration? Results of a geographical information system (GIS) approach for urban forestry research. Forest Policy and Economics, 6(1), 3-13. https://doi.org/10.1016/S1389-9341(02)00067-9.

Germenis, A. E. (2014). The hygiene hypothesis and evolutionary medicine. Archives of Hellenic Medicine/Arheia Ellenikes Iatrikes, 31(6).

Gose, M., Plachta-Danielzik, S., Willié, B., Johannsen, M., Landsberg, B., \& Müller, M. J. (2013). Longitudinal influences of neighbourhood built and social environment on children's weight status. International Journal of Environmental Research and Public Health, 10(10), 5083-5096. https://doi.org/10.3390/ ijerph10105083.

Goyder, E., Hind, D., Breckon, J., Dimairo, M., Minton, J., EversonHock, E., \& Cooper, C. (2014). A randomised controlled trial and cost-effectiveness evaluation of "booster" interventions to sustain increases in physical activity in middle-aged adults in deprived urban neighbourhoods. Health Technology Assessment, 18(13), 1-209. https://doi.org/10.3310/hta18130.

Graça, M., Queirós, C., Farinha-Marques, P., \& Cunha, M. (2018). Street trees as cultural elements in the city: Understanding how perception affects ecosystem services management in Porto. Portugal. Urban Forestry and Urban Greening, 30(February), 194-205. https://doi.org/10.1016/j.ufug.2018.02.001.

Grani, F., \& Bruun-Pedersen, J. R. (2017). Giro: Better biking in virtual reality. 2017 IEEE 3rd Workshop on Everyday Virtual Reality, WEVR 2017. https://doi.org/10.1109/WEVR.2017.7957711.

Grazuleviciene, R., Vencloviene, J., Kubilius, R., Grizas, V., Danileviciute, A., Dedele, A., \& Nieuwenhuijsen, M. J. (2016). Tracking restoration of park and urban street settings in coronary artery disease patients. International Journal of Environmental Research and Public Health, 13(6), 1-9. https://doi.org/10.3390/ ijerph13060550.

Green, O. O., Garmestani, A. S., Albro, S., Ban, N. C., Berland, A., Burkman, C. E., \& Shuster, W. D. (2016). Adaptive governance to promote ecosystem services in urban green spaces. Urban Ecosystems, 19(1), 77-93. https://doi.org/10.1007/s11252015-0476-2.

Grigsby-Toussaint, D. S., Turi, K. N., Krupa, M., Williams, N. J., PandiPerumal, S. R., \& Jean-Louis, G. (2015). Sleep insufficiency and the natural environment: Results from the US Behavioral Risk Factor Surveillance System survey. Preventive Medicine, 78, 78-84. https://doi.org/10.1016/j.ypmed.2015.07.011.

Guite, H. F., Clark, C., \& Ackrill, G. (2006). The impact of the physical and urban environment on mental well-being. Public Health, 120(12), 1117-1126. https://doi.org/10.1016/j.puhe.2006.10.005.

Haggag, M. A. (2010). The use of green walls in sustainable urban context: With reference to Dubai, UAE. WIT Transactions 
on Ecology and the Environment, 128, 261-270. https://doi. org/10.2495/ARC100221.

Hawkley, L. C., \& Cacioppo, J. T. (2007). Aging and loneliness: Downhill quickly? Current Directions in Psychological Science, 16(4), 187-191. https://doi.org/10.1111/j.1467-8721.2007.00501.

Health Evaluation and Applied Research Development (HEARD) (2018). Rapid reviews vs. systematic reviews: What are the differences? https://www.heardproject.org/news/rapid-review-vssystematic-review-what-are-the-differences.

Helbich, M. (2019). Dynamic Urban environmental exposures on Depression and Suicide (NEEDS) in the Netherlands: A protocol for a cross-sectional smartphone tracking study and a longitudinal population register study. BMJ Open, 9(8), 1-10. https://doi.org/10.1136/bmjopen-2019-030075.

Helbich, M., de Beurs, D., Kwan, M. P., O'Connor, R. C., \& Groenewegen, P. P. (2018). Natural environments and suicide mortality in the Netherlands: A cross-sectional, ecological study. The Lancet Planetary Health, 2(3), e134-e139. https:// doi.org/10.1016/S2542-5196(18)30033-0.

Helbich, M., van Emmichoven, M. J. Z., Dijst, M. J., Kwan, M. P., Pierik, F. H., \& Vries, S. I. D. (2016). Natural and built environmental exposures on children's active school travel: A Dutch global positioning system-based cross-sectional study. Health and Place, 39, 101-109. https://doi.org/10.1016/j. healthplace.2016.03.003.

Hermida, M. A., Neira, M., Cabrera-Jara, N., \& Osorio, P. (2017). Resilience in Latin American cities: Behaviour vs. space quality in the riverbanks of the Tomebamba River. Procedia Engineering, 198(September 2016), 467-481. https://doi.org/10.1016/j.proeng. 2017.07.101.

Hernandez, R. O. (2007). Effects of therapeutic gardens in special care units for people with dementia. Journal of Housing for the Elderly, 21(1-2), 153-177. https://doi.org/10.1300/J081v21n01.

Hillsdon, M., Panter, J., Foster, C., \& Jones, A. (2006). The relationship between access and quality of urban green space with population physical activity. Public Health, 120(12), 1127-1132. https://doi. org/10.1016/j.puhe.2006.10.007.

Hoffimann, E., Barros, H., \& Ribeiro, A. I. (2017). Socioeconomic inequalities in green space quality and Accessibility-evidence from a Southern European city. International Journal of Environmental Research and Public Health, 14(8). https://doi. org/10.3390/ijerph14080916.

Huang, N. C., Kung, S. F., \& Hu, S. C. (2018). The relationship between urbanization, the built environment, and physical activity among older adults in Taiwan. International Journal of Environmental Research and Public Health, 15(5), 1-20. https:// doi.org/10.3390/ijerph15050836.

Huang, W. Z., Yang, B. Y., Yu, H. Y., Bloom, M. S., Markevych, I., Heinrich, J., \& Dong, G. H. (2020). Association between community greenness and obesity in urban-dwelling Chinese adults. Science of the Total Environment, 702, 135040. https:// doi.org/10.1016/j.scitotenv.2019.135040.

Hui, N., Jumpponen, A., Francini, G., Kotze, D. J., Liu, X., Romantschuk, M., \& Setälä, H. (2017). Soil microbial communities are shaped by vegetation type and park age in cities under cold climate. Environmental Microbiology, 19(3), 1281-1295. https://doi. org/10.1111/1462-2920.13660.

Hunter, R. F., Cleland, C., Cleary, A., Droomers, M., Wheeler, B. W., Sinnett, D., \& Braubach, M. (2019). Environmental, health, wellbeing, social and equity effects of urban green space interventions: A meta-narrative evidence synthesis. Environment International, 130(June), 104923. https://doi.org/10.1016/j. envint.2019.104923.

Huynh, Q., Craig, W., Janssen, I., \& Pickett, W. (2013). Exposure to public natural space as a protective factor for emotional well-being among young people in Canada. BMC Public Health, 13(1). https://doi. org/10.1186/1471-2458-13-407.

Jamaludin, A. A., Keumala, N., Ariffin, A. R. M., \& Hussein, H. (2014). Landscape and sustainability: Three residential college buildings in the tropics. Open House International, 39(1), 92-106.

Jansen-Kosterink, S., Varenbrink, P., \& Naafs, A. (2018). The GezelschApp. A Dutch mobile application to reduce social isolation and loneliness. In ICT4AWE 2018 - Proceedings of the 4th International Conference on Information and Communication Technologies for Ageing Well and e-Health (Vol. 2018-March, pp. 142-147). https://doi.org/10.5220/0006732501420147.

Jansen, F. M., Ettema, D. F., Kamphuis, C. B. M., Pierik, F. H., \& Dijst, M. J. (2017). How do type and size of natural environments relate to physical activity behavior? Health and Place, 46(May), 73-81. https://doi.org/10.1016/j.healthplace.2017.05.005.

Janssen, I., \& Rosu, A. (2015). Undeveloped green space and freetime physical activity in 11 to 13 -year-old children. International Journal of Behavioral Nutrition and Physical Activity, 12(1), 4-10. https://doi.org/10.1186/s12966-015-0187-3.

Jim, C. Y., \& Chen, W. Y. (2007). Consumption preferences and environmental externalities: A hedonic analysis of the housing market in Guangzhou. Geoforum, 38(2), 414-431. https://doi. org/10.1016/j.geoforum.2006.10.002.

Jim, C. Y., \& Shan, X. (2013). Socioeconomic effect on perception of urban green spaces in Guangzhou, China. Cities, 31, 123-131. https://doi.org/10.1016/j.cities.2012.06.017.

Jones, K. R. (2018). 'The lungs of the city': Green space, public health and bodily metaphor in the landscape of urban park history. Environment and History, 24(1), 39-58. https://doi. org/10.3197/096734018X15137949591837.

Kabisch, N., van den Bosch, M., \& Lafortezza, R. (2017). The health benefits of nature-based solutions to urbanization challenges for children and the elderly - a systematic review. Environmental Research, 159(August), 362-373. https://doi.org/10.1016/j. envres.2017.08.004.

Kaplan, R., \& Kaplan, S. (1989). The experience of nature: A psychological perspective. Cambridge: Cambridge University Press.

Kessel, A., Green, J., Pinder, R., Wilkinson, P., Grundy, C., \& Lachowycz, K. (2009). Multidisciplinary research in public health: A case study of research on access to green space. Public Health, 123(1), 32-38. https://doi.org/10.1016/j. puhe.2008.08.005.

Khangura, S., Polisena, J., Clifford, T. J., Farrah, K., \& Kamel, C. (2014). Rapid review: An emerging approach to evidence synthesis in health technology assessment. International Journal Technology Assessment Health Care, 30(1), 20-7.

King, D. K., Litt, J., Hale, J., Burniece, K. M., \& Ross, C. (2015). "The park a tree built": Evaluating how a park development project impacted where people play. Urban Forestry and Urban Greening, 14(2), 293-299. https://doi.org/10.1016/j. ufug.2015.02.011.

King, T. L., Thornton, L. E., Bentley, R. J., \& Kavanagh, A. M. (2012). Does parkland influence walking? The relationship between area of parkland and walking trips in Melbourne, Australia. International Journal of Behavioral Nutrition and Physical Activity, 9, 1-9. https://doi.org/10.1186/1479-5868-9-115.

Kjellgren, A., \& Buhrkall, H. (2010). A comparison of the restorative effect of a natural environment with that of a simulated natural environment. Journal of Environmental Psychology, 30(4), 464472. https://doi.org/10.1016/j.jenvp.2010.01.011.

Kondo, M. C., South, E. C., Branas, C. C., Richmond, T. S., \& Wiebe, D. J. (2017). The association between urban tree cover and gun assault: A case-control and case-crossover study. American Journal of Epidemiology, 186(3), 289-296. https:// doi.org/10.1093/aje/kwx096. 
Kondo, M. C., Triguero-Mas, M., Donaire-Gonzalez, D., Seto, E., Valentín, A., Hurst, G., \& Nieuwenhuijsen, M. J. (2020). Momentary mood response to natural outdoor environments in four European cities. Environment International, 134(October 2019), 105237. https://doi.org/10.1016/j.envint.2019.105237.

Kruuse Afverchou, A. (2005). Green roofs, storm water management, and biodiversity in Malmö, Sweden. Transactions on Ecology and the Environment, 81, 171-179. Retrieved from http://cat. inist.fr/?aModele $=$ afficheN\&cpsidt $=17625960$.

Kumagai, Y., Gibson, R. B., \& Filion, P. (2015). Evaluating long-term urban resilience through an examination of the history of green spaces in Tokyo. Local Environment, 20(9), 1018-1039. https:// doi.org/10.1080/13549839.2014.887060.

Lachowycz, K., Jones, A. P., Page, A. S., Wheeler, B. W., \& Cooper, A. R. (2012). What can global positioning systems tell us about the contribution of different types of urban greenspace to children's physical activity? Health and Place, 18(3), 586-594. https://doi. org/10.1016/j.healthplace.2012.01.006.

Landeiro, F., Barrows, P., Nuttall Musson, E., Gray, A. M., \& Leal, J. (2017). Reducing social isolation and loneliness in older people: A systematic review protocol. BMJ Open, 7(5), 1-5. https://doi. org/10.1136/bmjopen-2016-013778.

Lanki, T., Siponen, T., Ojala, A., Korpela, K., Pennanen, A., Tiittanen, P., \& Tyrväinen, L. (2017). Acute effects of visits to urban green environments on cardiovascular physiology in women: A field experiment. Environmental Research, 159(July), 176-185. https:// doi.org/10.1016/j.envres.2017.07.039.

Lee, H. J., \& Lee, D. K. (2019). Do sociodemographic factors and urban green space affect mental health outcomes among the urban elderly population? International Journal of Environmental Research and Public Health, 16(5). https://doi.org/10.3390/ ijerph16050789.

Li, F., Hu, D., Wang, R., Yang, W., Paulussen, J., \& Liu, X. (2008). Comprehensive urban planning and management at multiple scales based on ecological principles: A case study in Beijing, China. International Journal of Sustainable Development and World Ecology, 15(6), 524-533. https://doi.org/10.1080/ 13504500809469848.

Li, X., \& Ghosh, D. (2018). Associations between body mass index and urban "Green" streetscape in Cleveland, Ohio, USA. International Journal of Environmental Research and Public Health, 15(10). https://doi.org/10.3390/ijerph15102186.

Liang, H., Chen, D., \& Zhang, Q. (2017). Walking accessibility of urban parks in a compact megacity. Proceedings of the Institution of Civil Engineers: Urban Design and Planning, 170(2), 59-71. https://doi.org/10.1680/jurdp.16.00030.

Liao, J., Yang, S., Xia, W., Peng, A., Zhao, J., Li, Y., \& Xu, S. (2019). Associations of exposure to green space with problem behaviours in preschool-aged children. International Journal of Epidemiology, 1-10. https://doi.org/10.1093/ije/dyz243.

Liao, J., Zhang, B., Xia, W., Cao, Z., Zhang, Y., Liang, S., \& Li, Y. (2019). Residential exposure to green space and early childhood neurodevelopment. Environment International, 128(October 2018), 70-76. https://doi.org/10.1016/j.envint.2019.03.070.

Liddicoat, C., Sydnor, H., Cando-Dumancela, C., Dresken, R., Liu, J., Gellie, N. J. C., \& Breed, M. F. (2020). Naturally-diverse airborne environmental microbial exposures modulate the gut microbiome and may provide anxiolytic benefits in mice. Science of the Total Environment, 701, 134684. https://doi.org/10.1016/j. scitotenv.2019.134684.

Lin, Y., Borghese, M. M., \& Janssen, I. (2018). Bi-directional association between sleep and outdoor active play among 10-13 year olds. BMC Public Health, 18(1), 1-8. https://doi. org/10.1186/s12889-018-5122-5.

Liu, W., Chen, W., \& Dong, C. (2017). Spatial decay of recreational services of urban parks: Characteristics and influencing factors.
Urban Forestry and Urban Greening, 25(May), 130-138. https:// doi.org/10.1016/j.ufug.2017.05.004.

Logan, A. C., Katzman, M. A., \& Balanzá-Martínez, V. (2015). Natural environments, ancestral diets, and microbial ecology: Is there a modern " paleo-deficit disorder"? Part I. Journal of Physiological Anthropology, 34(1), 1-18. https://doi.org/10. 1186/s40101-015-0041-y.

Ludden, G. D. S., van Rompay, T. J. L., Niedderer, K., \& Tournier, I. (2019). Environmental design for dementia care - towards more meaningful experiences through design. Maturitas, 128(March), 10-16. https://doi.org/10.1016/j.maturitas.2019.06.011.

Lyu, B., Zeng, C., Deng, S., Liu, S., Jiang, M., Li, N., \& Chen, Q. (2018). Bamboo forest therapy contributes to the regulation of psychological responses. Journal of Forest Research Vol 24, No 1. Journal of Forest Research.

Ma, B. D., Ng, S. L., Schwanen, T., Zacharias, J., Zhou, M., Kawachi, I., \& Sun, G. (2018). Pokémon GO and physical activity in Asia: Multilevel study. Journal of Medical Internet Research, 20(6), 1-12. https://doi.org/10.2196/jmir.9670.

Maas, J., van Dillen, S. M. E., Verheij, R. A., \& Groenewegen, P. P. (2009). Social contacts as a possible mechanism behind the relation between green space and health. Health and Place, 15(2), 586-595. https://doi.org/10.1016/j.healthplace.2008.09.006.

da Magalhães, A. P. T., \& F., Pina, M. de F. R. P. de, \& Ramos, E. da C. P. . (2017). The role of urban environment, social and health determinants in the tracking of leisure-time physical activity throughout adolescence. Journal of Adolescent Health, 60(1), 100-106. https://doi.org/10.1016/j.jadohealth.2016.08.015.

Mäki-Opas, T. E., Borodulin, K., Valkeinen, H., Stenholm, S., Kunst, A. E., Abel, T., \& Koskinen, S. (2016). The contribution of travel-related urban zones, cycling and pedestrian networks and green space to commuting physical activity among adults-a cross-sectional population-based study using geographical information systems. BMC Public Health, 16(1). https://doi. org/10.1186/s12889-016-3264-x.

Manferdelli, G., Torre, A. LA, \& Codella, R. (2019). Outdoor physical activity bears multiple benefits to health and society. The Journal of Sports Medicine and Physical Fitness 2019 Jan 16 - Minerva Medica - Journals. Retrieved from https://www. minervamedica.it/en/journals/sports-med-physical-fitness/article. php?cod=R40Y9999N00A19011601.

Marquet, O., Aaron Hipp, J., Alberico, C., Huang, J. H., Fry, D., Mazak, E., \& Floyd, M. F. (2019). Park use preferences and physical activity among ethnic minority children in low-income neighborhoods in New York City. Urban Forestry and Urban Greening, 38(January), 346-353. https://doi.org/10.1016/j. ufug.2019.01.018.

McGrath, L. J., Hopkins, W. G., \& Hinckson, E. A. (2015). Associations of objectively measured built-environment attributes with youth moderate-vigorous physical activity: A systematic review and meta-analysis. Sports Medicine, 45(6), 841-865. https://doi. org/10.1007/s40279-015-0301-3.

Michimi, A., \& Wimberly, M. C. (2012). Natural environments, obesity, and physical activity in nonmetropolitan areas of the United States. Journal of Rural Health, 28(4), 398-407. https:// doi.org/10.1111/j.1748-0361.2012.00413.x.

Mihrshahi, S., Gow, M. L., \& Baur, L. A. (2018). Contemporary approaches to the prevention and management of paediatric obesity: An Australian focus. Medical Journal of Australia, 209(6), 267-274. https://doi.org/10.5694/mja18.00140.

Milligan, C., Gatrell, A., \& Bingley, A. (2004). "Cultivating health": Therapeutic landscapes and older people in northern England. Social Science and Medicine, 58(9), 1781-1793. https://doi. org/10.1016/S0277-9536(03)00397-6.

Miralles-Guasch, C., Dopico, J., Delclòs-Alió, X., Knobel, P., Marquet, O., Maneja-Zaragoza, R., \& Vich, G. (2019). Natural landscape, 
infrastructure, and health: The physical activity implications of urban green space composition among the elderly. International Journal of Environmental Research and Public Health, 16(20). https://doi.org/10.3390/ijerph16203986.

Moffat, B. M., Johnson, J. L., \& Shoveller, J. A. (2009). A gateway to nature: Teenagers' narratives on smoking marijuana outdoors. Journal of Environmental Psychology, 29(1), 86-94. https://doi. org/10.1016/j.jenvp.2008.05.007.

Moher, D., Stewart, L., \& Shekelle, P. (2015). All in the family: Systematic reviews, rapid reviews, scoping reviews, realist reviews, and more. Systematic Review, 4, 183. https://doi. org/10.1186/s13643-015-0163-7.

Møller, M. S., Olafsson, A. S., Vierikko, K., Sehested, K., Elands, B., Buijs, A., \& van den Bosch, C. K. (2019). Participation through place-based e-tools: A valuable resource for urban green infrastructure governance? Urban Forestry and Urban Greening, 40, 245-253. https://doi.org/10.1016/j.ufug.2018.09.003.

Morris, B. L., Darling, W. G., Cronin, A. A., Rueedi, J., Whitehead, E. J., \& Gooddy, D. C. (2006). Assessing the impact of modern recharge on a sandstone aquifer beneath a suburb of Doncaster UK. Hydrogeology Journal, 14(6), 979-997. https://doi. org/10.1007/s10040-006-0028-1.

Mukherjee, D., Safraj, S., Tayyab, M., Shivashankar, R., Patel, S. A., Narayanan, G., \& Prabhakaran, D. (2017a). Park availability and major depression in individuals with chronic conditions: Is there an association in urban India? Health and Place, 47(May), 54-62. https://doi.org/10.1016/j.healthplace.2017.07.004.

Mukherjee, D., Safraj, S., Tayyab, M., Shivashankar, R., Patel, S. A., Narayanan, G., \& Prabhakaran, D. (2017b). Park availability and major depression in individuals with chronic conditions: Is there an association in urban India? Health and Place, 47(November 2016), 54-62. https://doi.org/10.1016/j.healthplace.2017.07.004.

Müller, G., Harhoff, R., Rahe, C., \& Berger, K. (2018). Inner-city green space and its association with body mass index and prevalent type 2 diabetes: A cross-sectional study in an urban German city. BMJ Open, 8(1), 1-9. https://doi.org/10.1136/bmjopen-2017019062.

Murphy, P. F., Miyazaki, Y., Detweiler, M. B., \& Kim, K. Y. (2010). Longitudinal analysis of differential effects on agitation of a therapeutic wander garden for dementia patients based on ambulation ability. Dementia, 9(3), 355-373. https://doi. org/10.1177/1471301210375336.

Mygind, L., Stevenson, M. P., Liebst, L. S., Konvalinka, I., \& Bentsen, P. (2018). Stress response and cognitive performance modulation in classroom versus natural environments: A quasi-experimental pilot study with children. International Journal of Environmental Research and Public Health, 15(6). https://doi.org/10.3390/ ijerph15061098.

Nakau, M., Imanishi, J., Imanishi, J., Watanabe, S., Imanishi, A., Baba, T., \& Morimoto, Y. (2013). Spiritual care of cancer patients by integrated medicine in urban green space: A pilot study. Explore: The Journal of Science and Healing, 9(2), 87-90. https://doi. org/10.1016/j.explore.2012.12.002.

Ng, E. \& Fisher, A. (2013). Understanding Well-Being in Multi-Levels: A review. Health, Culture and Society, 5. https://doi.org/10.5195/ hcs.2013.142.

Ngom, R., Gosselin, P., \& Blais, C. (2016). Reduction of disparities in access to green spaces: Their geographic insertion and recreational functions matter. Applied Geography, 66, 35-51. https://doi.org/10.1016/j.apgeog.2015.11.008.

Nichani, V., Dirks, K., Burns, B., Bird, A., \& Grant, C. (2017). Green space and depression during pregnancy: Results from the growing up in New Zealand study. International Journal of Environmental Research and Public Health, 14(9), 1-18. https:// doi.org/10.3390/ijerph14091083.
Noordzij, M. J., Beenackers, M. A., Oude Groeniger, J., \& Van Lenthe, F. J. (2020). Effect of changes in green spaces on mental health in older adults: A fixed effects analysis. Journal of Epidemiology and Community Health, 74(1), 48-56. https://doi.org/10.1136/ jech-2019-212704.

Nordbø, E. C. A., Raanaas, R. K., Nordh, H., \& Aamodt, G. (2019). Neighborhood green spaces, facilities and population density as predictors of activity participation among 8-year-olds: A crosssectional GIS study based on the Norwegian mother and child cohort study. BMC public health, 19(1), 1426.

Nutsford, D., Pearson, A. L., \& Kingham, S. (2013). An ecological study investigating the association between access to urban green space and mental health. Public Health, 127(11), 1005-1011. https://doi.org/10.1016/j.puhe.2013.08.016.

Occhiuto, R. (2018). Resistance \& permanence of green urban systems in the globalization age, (September), 1711-1718. https://doi. org/10.4995/isuf2017.2017.6328.

OECD. (2013). OECD Guidelines on Measuring Subjective Wellbeing. OECD Publishing, Paris,. https://doi.org/10.1787/ 9789264191655-en.

OECD (2019a), How's Life in the Digital Age?: Opportunities and Risks of the Digital Transformation for People's Wellbeing, https:// doi.org/10.1787/9789264311800-en.

Ord, K., Mitchell, R., \& Pearce, J. (2017). Is level of neighbourhood green space associated with physical activity in green space? PubMed - NCBI, 1-8. Retrieved from https://www-ncbi-nlmnih-gov.proxy.library.cornell.edu/pubmed/24219824.

Othman, R., Abu Kasim, S. Z., Hashim, K. S. H. Y., \& Mohd Noor, N. F. (2015). Novel landscape design model system approach: Monitoring, predicting and calculating carbon sequestration rate for tourism accommodation premises green spaces. Journal Teknologi, 77(30), 89-99. https://doi.org/10.11113/jt.v77.6872.

Panyadee, P., Balslev, H., Wangpakapattanawong, P., \& Inta, A. (2016). Woody plant diversity in urban homegardens in Northern Thailand. Economic Botany, 70(3), 285-302. https://doi. org/10.1007/s12231-016-9348-9.

Paquet, C., Orschulok, T. P., Coffee, N. T., Howard, N. J., Hugo, G., Taylor, A. W., \& Daniel, M. (2013). Are accessibility and characteristics of public open spaces associated with a better cardiometabolic health? Landscape and Urban Planning, 118, 70-78. https://doi.org/10.1016/j.landurbplan.2012.11.011.

Park, S. (2017). A preliminary study on connectivity and perceived values of community green spaces. Sustainability (Switzerland), 9(5). https://doi.org/10.3390/su9050692.

Patel, D. M., Block, R. C., Chapman, B. P., Korfmacher, K. S., \& van Wijngaarden, E. (2019). Green space and mental health symptoms in a cardiac rehabilitation population. Indoor and Built Environment, 28(10), 1431-1440. https://doi.org/10.1177/ 1420326X19853615.

Pereira, M., Nogueira, H., \& Padez, C. (2019). The role of urban design in childhood obesity: A case study in Lisbon. Portugal. American Journal of Human Biology, 31(3), 1-9. https://doi.org/10.1002/ ajhb. 23220 .

Polisena, J., Garritty, C., Kamel, C., Stevens, A., \& Abou-Setta, A. M. (2015). Rapid review programs to support health care and policy decision making: A descriptive analysis of processes and methods. Systematic Review, 4, 26. https://doi.org/10.1186/s13643015-0022-6.

Potestio, M. L., Patel, A. B., Powell, C. D., McNeil, D. A., Jacobson, R. D., \& McLaren, L. (2009). Is there an association between spatial access to parks/green space and childhood overweight/ obesity in Calgary, Canada? International Journal of Behavioral Nutrition and Physical Activity, 6, 1-10. https://doi. org/10.1186/1479-5868-6-77.

Pourzargar, M. R. (2016). Regeneration and restoration of old texture with a perspective to protect the identity of housing, case 
study-Joybareh locality in Isfahan *. Architecture \& Urbanism, 12(37), 41-52.

Prince, S. A., Kristjansson, E. A., Russell, K., Billette, J. M., Sawada, M., Ali, A., \& Prud'homme, D. (2011). A multilevel analysis of neighbourhood built and social environments and adult self-reported physical activity and body mass index in Ottawa, Canada. International Journal of Environmental Research and Public Health, 8(10), 3953-3978. https://doi.org/10.3390/ ijerph8103953.

Puhakka, S., Pyky, R., Lankila, T., Kangas, M., Rusanen, J., Ikäheimo, T. M., \& Korpelainen, R. (2018). Physical activity, residential environment, and nature relatedness in young men-a population-based MOPO study. International Journal of Environmental Research and Public Health, 15(10), 1-12. https:// doi.org/10.3390/ijerph15102322.

Pun, V. C., Manjourides, J., \& Suh, H. H. (2018). Association of neighborhood greenness with self-perceived stress, depression and anxiety symptoms in older U.S adults. Environmental Health: A Global Access Science Source, 17(1), 1-11. https:// doi.org/10.1186/s12940-018-0381-2.

Rahman, K. M. A., \& Zhang, D. (2018). Analyzing the level of accessibility of public urban green spaces to different socially vulnerable groups of people. Sustainability (Switzerland), 10(11). https://doi.org/10.3390/su10113917.

Rahman, M. M., Rahman, M. M., \& Momotaz, M. (2019). Environmental quality evaluation in Dhaka City CorporationUsing satellite imagery. Proceedings of the Institution of Civil Engineers: Urban Design and Planning, 172(1), 13-25. https:// doi.org/10.1680/jurdp.17.00032.

Raymond, C. M., Gottwald, S., Kuoppa, J., \& Kyttä, M. (2016). Integrating multiple elements of environmental justice into urban blue space planning using public participation geographic information systems. Landscape and Urban Planning, 153, 198208. https://doi.org/10.1016/j.landurbplan.2016.05.005.

Reid, C. E., O’Neill, M. S., Gronlund, C. J., Brines, S. J., Brown, D. G., Diez-Roux, A. V., \& Schwartz, J. (2009). Mapping community determinants of heat vulnerability. Environmental Health Perspectives, 117(11), 1730-1736. https://doi.org/10.1289/ehp. 0900683.

Ribeiro, A. I., De Fátima De Pina, M., \& Mitchell, R. (2015). Development of a measure of multiple physical environmental deprivation. After United Kingdom and New Zealand, Portugal. European Journal of Public Health, 25(4), 610-617. https://doi. org/10.1093/eurpub/cku242.

Ribeiro, A. I., Tavares, C., Guttentag, A., \& Barros, H. (2019). Association between neighbourhood green space and biological markers in school-aged children. Findings from the Generation XXI birth cohort. Environment International, 132(July), 105070. https://doi.org/10.1016/j.envint.2019.105070.

Richardson, A. S., Troxel, W. M., Ghosh-Dastidar, M. B., Beckman, R., Hunter, G. P., DeSantis, A. S., \& Dubowitz, T. (2017). One size doesn't fit all: Cross-sectional associations between neighborhood walkability, crime and physical activity depends on age and sex of residents. BMC Public Health, 17(1), 1-11. https://doi.org/10.1186/s12889-016-3959-z.

Ring, L., Barry, B., Totzke, K., \& Bickmore, T. (2013). Addressing loneliness and isolation in older adults: Proactive affective agents provide better support. In Proceedings - 2013 Humaine Association Conference on Affective Computing and Intelligent Interaction, ACII 2013. https://doi.org/10.1109/ACII.2013.17.

Rook, G. A. (2013). Regulation of the immune system by biodiversity from the natural environment: An ecosystem service essential to health. Proceedings of the National Academy of Sciences, 110(46), 18360-18367. https://doi.org/10.1073/pnas. 1313731110 .
Rook, G. A. W., Raison, C. L., \& Lowry, C. A. (2014). Microbial "old friends", immunoregulation and socioeconomic status. Clinical and Experimental Immunology, 177(1), 1-12. https:// doi.org/10.1111/cei.12269.

Rudnev, V. (2012). The factor of local cultural specificity and process of globalization. Collegium Antropologicum, 36(4), 1135-1138.

Saghafi, D. M., \& Ahmadpour, S. (2017). Investigation into structural formation of social relations of the elderly (case study, Golsar Vicinity, Rasht, Iran). Australian Ranger Bulletin, 4(1), 9-10.

Sanchez, C. A., Thomas, K. E., Malilay, J., \& Annest, J. L. (2010). Environmental injuries treated, 33(1), 3-10.

Servadio, J. L., Lawal, A. S., Davis, T., Bates, J., Russell, A. G., Ramaswami, A., \& Botchwey, N. (2019). Demographic inequities in health outcomes and air pollution exposure in the Atlanta area and its relationship to urban infrastructure. Journal of Urban Health, 96(2), 219-234. https://doi.org/10.1007/s11524018-0318-7.

Shackleton, S., Chinyimba, A., Hebinck, P., Shackleton, C., \& Kaoma, H. (2015). Multiple benefits and values of trees in urban landscapes in two towns in northern South Africa. Landscape and Urban Planning, 136, 76-86. https://doi.org/10.1016/j. landurbplan.2014.12.004.

Shourbela, R. M., El-latif, A. M. A., \& El-Gawad, E. A. A. (2016). The latest status and distribution of fishes in upper tigris river and two new records for Turkish Freshwaters Cüneyt. Turkish Journal of Fisheries and Aquatic Sciences, 16, 651-657. https:// doi.org/10.4194/1303-2712-v16.

Shrestha, S. K., Katelaris, C., Dharmage, S. C., Burton, P., Vicendese, D., Tham, R., \& Erbas, B. (2018). High ambient levels of grass, weed and other pollen are associated with asthma admissions in children and adolescents: A large 5-year case-crossover study. Clinical and Experimental Allergy, 48(11), 1421-1428. https:// doi.org/10.1111/cea.13225.

Simon, N., Saxe, G. N., \& Marmar, C. R. (2020). Mental health disorders related to COVID-19-related deaths. Journal of the American Medical Association, 324(15), 1493-1494. https://doi. org/10.1001/jama.2020.19632.

Son, J.-Y., Liu, J. C., \& Bell, M. L. (2019). Temperature-related mortality: A systematic review and investigation of effect modifiers. Environmental Research Letters, 14(7), 073004. https:// doi.org/10.1088/1748-9326/ab1cdb.

Sorkin, D., Rook, K. S., \& Lu, J. L. (2002). Loneliness, lack of emotional support, lack of companionship, and the likelihood of having a heart condition in an elderly sample. Annals of Behavioral Medicine, 24(4), 290-298. https://doi.org/10.1207/ S15324796ABM2404_05.

Stewart, O. T., Moudon, A. V., Littman, A. J., Seto, E., \& Saelens, B. E. (2018). Why neighborhood park proximity is not associated with total physical activity. Health and Place, 52(May), 163-169. https://doi.org/10.1016/j.healthplace.2018.05.011.

Stewart, O. T., Moudon, A. V., Littman, A. J., Seto, E., \& Saelens, B. E. (2018b). Why neighborhood park proximity is not associated with total physical activity. Health and Place, 52(November 2017), 163-169. https://doi.org/10.1016/j. healthplace.2018.05.011.

Storgaard, R. L., Hansen, H. S., Aadahl, M., \& Glümer, C. (2013). Association between neighbourhood green space and sedentary leisure time in a Danish population, (July), 846-852. https://doi. org/10.1177/1403494813499459.

Su, J. G., Dadvand, P., Nieuwenhuijsen, M. J., Bartoll, X., \& Jerrett, M. (2019). Associations of green space metrics with health and behavior outcomes at different buffer sizes and remote sensing sensor resolutions. Environment International, 126(October 2018), 162-170. https://doi.org/10.1016/j.envint.2019.02.008.

Sugiyama, T., Paquet, C., Howard, N. J., Coffee, N. T., Taylor, A. W., Adams, R. J., \& Daniel, M. (2014). Public open spaces and 
walking for recreation: Moderation by attributes of pedestrian environments. Preventive Medicine, 62, 25-29. https://doi. org/10.1016/j.ypmed.2014.01.030.

Sugiyama, T., Villanueva, K., Knuiman, M., Francis, J., Foster, S., Wood, L., \& Giles-Corti, B. (2016). Can neighborhood green space mitigate health inequalities? A study of socio-economic status and mental health. Health and Place, 38, 16-21. https:// doi.org/10.1016/j.healthplace.2016.01.002.

Sun, Y., Du, Y., Wang, Y., \& Zhuang, L. (2017). Examining associations of environmental characteristics with recreational cycling behaviour by street-level strava data. International Journal of Environmental Research and Public Health, 14(6). https://doi.org/10.3390/ijerph14060644.

Tan, J., Zheng, Y., Song, G., Kalkstein, L. S., Kalkstein, A. J., \& Tang, X. (2007). Heat wave impacts on mortality in Shanghai, 1998 and 2003. International Journal of Biometeorology, 51(3), 193-200. https://doi.org/10.1007/s00484-006-0058-3.

Ten Bruggencate, T., Luijkx, K. G., \& Sturm, J. (2018). When your world gets smaller: How older people try to meet their social needs, including the role of social technology. Ageing and Society, 1-27. https://doi.org/10.1017/s0144686x18000260.

Tian, Y., \& Jim, C. Y. (2012). Development potential of sky gardens in the compact city of Hong Kong. Urban Forestry and Urban Greening, 11(3), 223-233. https://doi.org/10.1016/j. ufug.2012.03.003.

Tian, Y., Jim, C. Y., \& Wang, H. (2014). Assessing the landscape and ecological quality of urban green spaces in a compact city. Landscape and Urban Planning, 121, 97-108. https://doi. org/10.1016/j.landurbplan.2013.10.001.

Tikka, P. M., Kuitunen, M. T., \& Tynys, S. M. (2000). Effects of educational background on students' attitudes, Activity Levels, and Knowledge Concerning the Environment.

Triguero-Mas, M., Donaire-Gonzalez, D., Seto, E., Valentín, A., Martínez, D., Smith, G., \& Nieuwenhuijsen, M. J. (2017). Natural outdoor environments and mental health: Stress as a possible mechanism. Environmental Research, 159(April), 629-638. https://doi.org/10.1016/j.envres.2017.08.048.

Uitto, A., Juuti, K., Lavonen, J., \& Meisalo, V. (2006). Students' interest in biology and their out-of-school experiences. Journal of Biological Education, 40(3), 124-129. https://doi. org/10.1080/00219266.2006.9656029.

Van den Berg, A. E., Joye, Y., \& Koole, S. L. (2016). Why viewing nature is more fascinating and restorative than viewing buildings: A closer look at perceived complexity. Urban Forestry and Urban Greening. https://doi.org/10.1016/j.ufug.2016.10.011.

Van den Bosch, M. A., Östergren, P. O., Grahn, P., Skärbäck, E., \& Währborg, P. (2015). Moving to serene nature may prevent poor mental health-results from a swedish longitudinal cohort study. International Journal of Environmental Research and Public Health, 12(7), 7974-7989. https://doi.org/10.3390/ ijerph120707974.

Van Houwelingen-Snippe, J., van Rompay, T. J. L., \& Ben Allouch, S. (2020a). Feeling connected after experiencing digital nature: A survey study. International Journal of Environmental Research and Public Health, 17(18), 6879-6895.

Van Houwelingen-Snippe, J., van Rompay, T. J. L., de Jong, M. D. T., $\&$ Ben Allouch, S. (2020b). Does digital nature enhance social aspirations? An experimental study. International Journal of Environmental Research and Public Health, 17(4), 1454-1472.

Veitch, J., Carver, A., Abbott, G., Giles-Corti, B., Timperio, A., \& Salmon, J. (2015). How active are people in metropolitan parks? An observational study of park visitation in Australia. BMC Public Health, 15(1), 1-8. https://doi.org/10.1186/s12889015-1960-6.

Vienneau, D., de Hoogh, K., Faeh, D., Kaufmann, M., Wunderli, J. M., \& Röösli, M. (2017). More than clean air and tranquillity:
Residential green is independently associated with decreasing mortality. Environment International, 108(July), 176-184. https:// doi.org/10.1016/j.envint.2017.08.012.

Villeneuve, P. J., Jerrett, M., Su, G., \& J., Burnett, R. T., Chen, H., Wheeler, A. J., \& Goldberg, M. S. . (2012). A cohort study relating urban green space with mortality in Ontario. Canada. Environmental Research, 115(2012), 51-58. https://doi. org/10.1016/j.envres.2012.03.003.

Vogt, S., Mielck, A., Berger, U., Grill, E., Peters, A., Döring, A., \& Maier, W. (2015). Neighborhood and healthy aging in a German city: Distances to green space and senior service centers and their associations with physical constitution, disability, and healthrelated quality of life. European Journal of Ageing, 12(4), 273283. https://doi.org/10.1007/s10433-015-0345-0.

Wang, D., Lau, K. K. L., Yu, R., Wong, S. Y. S., Kwok, T. T. Y., \& Woo, J. (2017). Neighbouring green space and mortality in community-dwelling elderly Hong Kong Chinese: A cohort study. BMJ Open, 7(7), 1-10. https://doi.org/10.1136/bmjopen2016-015794.

Wang, K., \& Liu, J. (2017). The spatiotemporal trend of city parks in Mainland China between 1981 and 2014: Implications for the promotion of leisure time physical activity and planning. International Journal of Environmental Research and Public Health, 14(10). https://doi.org/10.3390/ijerph14101150.

Wang, Lan, Sun, W., Zhou, K., Zhang, M., \& Bao, P. (2019). Spatial analysis of built environment risk for respiratory health and its implication for urban planning: A case study of shanghai. International Journal of Environmental Research and Public Health, 16(8). https://doi.org/10.3390/ijerph16081455.

Wang, Lan, Zhao, X., Xu, W., Tang, J., \& Jiang, X. (2016). Correlation analysis of lung cancer and urban spatial factor: Based on survey in Shanghai. Journal of Thoracic Disease, 8(9), 2626-2637. https://doi.org/10.21037/jtd.2016.09.10.

Wang, Linwen, Chen, W., \& dong, \& Li, L. (2017). Investigation of the optical and electrical properties of $\mathrm{ZnO} / \mathrm{Cu} / \mathrm{ZnO}$ multilayer structure for transparent conductive electrodes by magnetron sputtering. Journal of Materials Science: Materials in Electronics, 28(4), 3458-3466. https://doi.org/10.1007/s10854016-5943-x.

Wang, Z., Qin, Z., He, J., Ma, Y., Ye, Q., Xiong, Y., \& Xu, F. (2019). The association between residential density and physical activity among urban adults in regional China. BMC Public Health, 19(1), 1-7. https://doi.org/10.1186/s12889-019-7593-4.

Wen, C., Albert, C., \& Von Haaren, C. (2018). The elderly in green spaces: Exploring requirements and preferences concerning nature-based recreation. Sustainable Cities and Society, 38(January), 582-593. https://doi.org/10.1016/j.scs.2018.01.023.

White, M. P., Yeo, N. L., Vassiljev, P., Lundstedt, R., Wallergård, M., Albin, M., \& Lõhmus, M. (2018). A prescription for "nature"- the potential of using virtual nature in therapeutics. Neuropsychiatric Disease and Treatment, 14, 3001-3013. https:// doi.org/10.2147/NDT.S179038.

Xie, T., Wang, M., Chen, W., \& Uwizeyimana, H. (2018). Impacts of urbanization and landscape patterns on the earthworm communities in residential areas in Beijing. Science of the Total Environment, 626, 1261-1269. https://doi.org/10.1016/j.scitotenv.2018.01.187.

$\mathrm{Xu}, \mathrm{D}$., \& Gao, J. (2017). The night light development and public health in China. Sustainable Cities and Society, 35(July), 57-68. https:// doi.org/10.1016/j.scs.2017.07.009.

Xu, N., Wang, J., \& Wang, W. (2019). Revealing urban public space patterns through quantitative comparison between the old city of Nanjing and Zurich. Sustainability (Switzerland), 11(13). https:// doi.org/10.3390/su11133687.

Younan, D., Li, L., Tuvblad, C., Wu, J., Lurmann, F., Franklin, M., \& Chen, J. C. (2018). Long-term ambient temperature and externalizing behaviors in adolescents. American Journal of 
Epidemiology, 187(9), 1931-1941. https://doi.org/10.1093/aje/ kwy104.

Younan, D., Tuvblad, C., Li, L., Wu, J., Lurmann, F., Franklin, M., \& Chen, J. (2016). Environmental determinants of aggression in adolescents. Journal of the American Academy of Child \& Adolescent Psychiatry, 55(7), 591-601. https://doi.org/10.1016/j. jaac.2016.05.002.

Zacharias, J., Sun, Z., Chuang, L., \& Lee, F. (2015). The hutong urban development model compared with contemporary suburban development in Beijing. Habitat International, 49, 260-265. https:// doi.org/10.1016/j.habitatint.2015.05.035.

Zamir, S., Hennessy, C. H., Taylor, A. H., \& Jones, R. B. (2018). Video-calls to reduce loneliness and social isolation within care environments for older people: An implementation study using collaborative action research. BMC Geriatrics, 18(1), 1-13. https:// doi.org/10.1186/s12877-018-0746-y.

Zandieh, R., Martinez, J., \& Flacke, J. (2019). Older adults' outdoor walking and inequalities in neighbourhood green spaces characteristics. International Journal of Environmental Research and Public Health, 16(22). https://doi.org/10.3390/ijerph16224379.

Zhang, C. J. P., Barnett, A., Johnston, J. M., Lai, P. C., Lee, R. S. Y., Sit, C. H. P., \& Cerin, E. (2019). Objectively-measured neighbourhood attributes as correlates and moderators of quality of life in older adults with different living arrangements: The alecs cross-sectional study. International Journal of Environmental Research and Public Health, 16(5), 1-19. https://doi.org/10.3390/ijerph16050876.

Zhang, Y., van Dijk, T., Tang, J., \& van den Berg, A. E. (2015). Green space attachment and health: A comparative study in two urban neighborhoods. International Journal of Environmental Research and Public Health, 12(11), 14342-14363. https://doi.org/10.3390/ ijerph121114342.

Zhu, L., \& Jia, Y. (2012). Construction of green space system of disaster-prevention in old districts of Zhengzhou city. Advanced Materials Research. https://doi.org/10.4028/www.scientific.net/ AMR.450-451.1314.

Zijlema, W. L., Triguero-Mas, M., Smith, G., Cirach, M., Martinez, D., Dadvand, P., \& Julvez, J. (2017). The relationship between natural outdoor environments and cognitive functioning and its mediators. Environmental Research, 155(November 2016), 268-275. https:// doi.org/10.1016/j.envres.2017.02.017.

Publisher's Note Springer Nature remains neutral with regard to jurisdictional claims in published maps and institutional affiliations. 